\title{
Logarithmic corrections to extremal black hole entropy from quantum entropy function
}

\author{
Shamik Banerjee, ${ }^{a}$ Rajesh K. Gupta ${ }^{a}$ and Ashoke Sen ${ }^{a, b}$ \\ ${ }^{a}$ Harish-Chandra Research Institute, \\ Chhatnag Road, Jhusi, Allahabad 211019, India \\ ${ }^{b}$ LPTHE, Universite Pierre et Marie Curie, Paris 6, \\ 4 Place Jussieu, 75252 Paris Cedex 05, France \\ E-mail: bshamik@mri.ernet.in, rajesh@mri.ernet.in, sen@mri.ernet.in
}

ABSTRACT: We evaluate the one loop determinant of matter multiplet fields of $\mathcal{N}=4$ supergravity in the near horizon geometry of quarter BPS black holes, and use it to calculate logarithmic corrections to the entropy of these black holes using the quantum entropy function formalism. We show that even though individual fields give non-vanishing logarithmic contribution to the entropy, the net contribution from all the fields in the matter multiplet vanishes. Thus logarithmic corrections to the entropy of quarter BPS black holes, if present, must be independent of the number of matter multiplet fields in the theory. This is consistent with the microscopic results. During our analysis we also determine the complete spectrum of small fluctuations of matter multiplet fields in the near horizon geometry.

KEYwords: Black Holes in String Theory, AdS-CFT Correspondence

ARXIV EPRINT: 1005.3044 


\section{Contents}

1 Introduction

2 Logarithmic correction to the black hole entropy due to a single scalar field

3 Heat kernels of vector, $p$-form and fermion fields 12

$\begin{array}{ll}3.1 \text { Vector fields } & 12 \\ 3.2 & - \text {-form }\end{array}$

$\begin{array}{lll}3.2 & p \text {-form fields } & 15\end{array}$

$\begin{array}{lll}3.3 & \text { Fermions } & 17\end{array}$

4 Effect of graviphoton background in $\mathcal{N}=4$ supersymmetric string theory

4.1 Four dimensional $\mathcal{N}=4$ supergravity from ten dimensional $\mathcal{N}=1$ supergravity

4.2 The quadratic action for the fluctuations around the attractor geometry

5 Eigenvalues, heat kernel and one loop effective action in the matter sector

6 Discussion

A Analysis of the zero mode contribution

\section{Introduction}

Wald's formula gives a generalization of the Bekenstein-Hawking entropy in a classical theory of gravity with higher derivative terms, possibly coupled to other matter fields [14]. In the extremal limit this leads to a simple algebraic procedure for determining the near horizon field configurations and the entropy [5, 6], leading to a simple proof of the attractor mechanism [7-9] in a general higher derivative theory coupled to matter.

Given this success, one could ask: is there a generalization of the Wald's formula to the full quantum theory? At least for extremal BPS black holes, there is reason to expect that such a formula might exist, since on the microscopic side there is a precise result for the degeneracy (more precisely an appropriate index) ${ }^{1}$ for these BPS black holes. One naive approach to this problem will be to continue to use Wald's formula by replacing the classical action by the one particle irreducible (1PI) action. In string theory this approach

\footnotetext{
${ }^{1}$ See ref. $[10,11]$ for a discussion on how the black hole entropy can be related to an index. In this paper we shall not distinguish between index and degeneracy.
} 
has been successful in a number of cases, producing highly non-trivial dependence of the entropy on the charges which can then be verified by explicit computation of the statistical entropy in a microscopic description [12-15]. (Further results on the microscopic spectrum of $\mathcal{N}=4$ supersymmetric string theories, which will be our focus of attention, can be found in [16-42]. Early studies on the macroscopic entropy of these black holes can be found in [43-48]).

There is however a simple reason why this cannot be the complete prescription. In its original formulation, Wald's formula holds only for local action. On the other hand the 1PI action at sufficiently high orders in derivatives contains non-local terms due to the presence of the massless fields in the supergravity theory. Thus the prescription of replacing the classical action by the 1PI action in Wald's formula cannot be the complete story. ${ }^{2}$ A proposal for overcoming this difficulty based on a Euclidean path integral approach was suggested in [50]. In this formulation, called the quantum entropy function formalism, the degeneracy associated with the black hole horizon is given by the finite part of the string theory partition function in the near horizon geometry of the black hole containing an $A d S_{2}$ factor. More precisely, the partition function is calculated by evaluating the string theory path integral over all string field configurations subject to the condition that near the asymptotic boundary of $A d S_{2}$ the configuration approaches the near horizon geometry of the extremal black hole under consideration. Such a partition function is divergent due to the infinite volume of $A d S_{2}$, but the rules of $A d S_{2} / C F T_{1}$ correspondence gives a precise procedure for removing this divergence. ${ }^{3}$ While in the classical limit this prescription gives us back the exponential of the Wald action, it can in principle be used to systematically calculate the quantum corrections to the entropy of an extremal black hole. Indeed many of the non-perturbative features of the known spectrum of quarter BPS states in $\mathcal{N}=4$ supersymmetric string theories have been reproduced from the macroscopic side using this formalism $[10,40,41,54-56]$. These non-perturvative effects arise from inclusion in the path integral the contribution from non-trivial saddle points which have the same asymptotic geometry as the near horizon geometry of the black hole, but differ from it in the interior of $A d S_{2}$.

In order to make full use of this program we need to carry out the path integral over the string fields around each saddle point. We can take two approaches to this problem. The simplicity of the microscopic formula for the black hole entropy in $\mathcal{N}=4$ and $\mathcal{N}=$ 8 supersymmetric string theories leads us to expect that the contribution to the path integral from each saddle point can be expressed as a finite dimensional integral with simple integrand. Given the large amount of supersymmetry possessed by the near horizon geometry, one could try to achieve this using localization techniques [57-68]. In particular it is quite conceivable that supersymmetry will help us localize the path integral over string fields to a finite dimensional subspace of the full configuration space, which could then be directly compared with the corresponding contribution to the microscopic formula [69]. On the other hand one could also take a brute force approach and try to evaluate the path

\footnotetext{
${ }^{2}$ See [49] for an attempt to resolve this using an auxiliary scalar field.

${ }^{3}$ Technically this is identical to the procedure one follows for removing the quark self-energy divergence while computing the Wilson /'t Hooft line expectation values in gauge theories via holographic method [5153], but whether there is a deeper physical connection between these two quantities remains to be seen.
} 
integral over string fields in perturbation theory around each saddle point. This can then be compared with a similar expansion of the microscopic degeneracy formula in appropriate inverse powers of the charges.

The analysis of this paper will be based on the second approach. We shall calculate the one loop contribution to the quantum entropy function to analyze one specific feature of the entropy formula, - logarithmic corrections to the classical entropy. More precisely, we shall consider the limit in which all charges become uniformly large, carrying a common scale $\Lambda$, and study corrections of order $\ln \Lambda$ to the entropy. ${ }^{4}$ The motivation for this study comes from the known results on the microscopic spectrum of the quarter BPS dyons in $\mathcal{N}=4$ supersymmetric string theories and $1 / 8$ BPS dyons in $\mathcal{N}=8$ supersymmetric string theories. If we denote by $\Delta$ the unique quartic combination of the charges which is invariant under continuous U-duality group of these theories, then for large $\Delta$ the microscopic entropy $S_{\text {micro }}$, computed by taking the logarithm of the appropriate helicity trace index [73, 74], grows as

$$
S_{\text {micro }}=\left\{\begin{array}{l}
\pi \sqrt{\Delta}+\mathcal{O}(1) \text { for } \mathcal{N}=4 \\
\pi \sqrt{\Delta}-2 \ln \Delta+\mathcal{O}(1) \text { for } \quad \mathcal{N}=8
\end{array} .\right.
$$

The result for the $\mathcal{N}=4$ theory can be found in $[12,13,15]$ and that for $\mathcal{N}=8$ theory can be found in [56]. Thus in the limit described above the quarter BPS dyons in $\mathcal{N}=4$ supersymmetric theories have no logarithmic corrections whereas $1 / 8$ BPS dyons in $\mathcal{N}=8$ supersymmetrc theories have corrections of order $-8 \ln \Lambda$. Our goal will be to understand some aspects of these results from the macroscopic viewpoint.

We shall begin by trying to understand the origin of possible logarithmic corrections to the entropy in the quantum entropy function formalism. As we shall see, for this study the contribution from the stringy modes - and the Kaluza-Klein modes associated with the internal directions - become irrelevant, and we only need to compute the contribution from the massless modes living on the near horizon $A d S_{2} \times S^{2}$ geometry. As a simple exercise we first calculate the one loop determinant of a massless scalar field in the near horizon $A d S_{2} \times S^{2}$ background using heat kernel method, ${ }^{5}$ and show that after following the prescription of extracting the entropy from the one loop partition function, we do generate a logarithmic correction to the entropy. Furthermore this agrees with earlier result of [77] calculated using a somewhat different approach (more detailed discussion on the comparison with other approaches will be given below).

Applying this procedure to compute logarithmic corrections to string theoretic black holes requires us to evaluate the one loop contribution to the partition function from the fluctuation of massless fields in the attractor geometry. The main technical difficulty in this computation is the diagonalization of the kinetic terms of various fields. Since the background contains electric and magnetic fields besides gravity, and since the supergravity action is non-linear, the fluctuations of scalars, vectors and metric (and similarly of spin $1 / 2$

\footnotetext{
${ }^{4}$ This is to be distinguished from the Cardy limit in which one of the charges representing momentum along an internal circle becomes large. In this limit the logarithmic correction to the black hole entropy is known to be universal [70-72].

${ }^{5}$ Similar calculations in $A d S_{3}$ background, with a somewhat different application in mind, can be found in $[75,76]$.
} 
and spin $3 / 2$ fields) mix with each other. However for quarter BPS black holes in $\mathcal{N}=4$ supersymmetric string theories, which will be the main focus of our analysis, there is a simplification: the near horizon background involves purly gravitational and graviphoton fields, but no matter multiplet fields. Due to this property the quadratic terms in the action expanded around this background do not contain any mixing term between matter and gravity multiplet fields. This allows us to analyze the contribution to the partition function from the matter multiplet fields and gravity multiplet fields separately.

In this paper we study the contribution to the partition function due to the fluctuations of the matter multiplets. The first step in this process is to find explicitly all the eigenvalues and eigenfunctions of the kinetic operator acting on the matter multiplet, both in the bosonic and the fermionic sectors. We then express the one loop contribution to the partition function in terms of this data, and find that in the final expression the contribution from the bosonic and the fermionic fields cancel. While a similar calculation is possible in principle for the fields in the gravitational sector, the computation is technically involved, and we have not carried out this analysis. As a result for any single theory we cannot make a definite macroscopic prediction for the black hole entropy. However if we consider a collection of different $\mathcal{N}=4$ supersymmetric string theories then our result has a definite prediction, namely that the logarithmic correction to the black hole entropy in $\mathcal{N}=4$ supersymmetric string theories is independent of the number of matter multiplets we have in the theory. This is borne out in the microscopic analysis, - the net logarithmic correction being zero in all known $\mathcal{N}=4$ supersymmetric string theories irrespective of the number of matter multiplets the theory contains $[12-15]$. In order to fully reproduce the results given in (1.1) we shall have to compute the contribution from the gravity multiplet in $\mathcal{N}=4$ and $\mathcal{N}=8$ supersymmetric string theories.

To put our results in context we note that part of the one loop contribution to the entropy of BPS black holes has been analyzed earlier, leading to non-trivial agreement between the microscopic and the macroscopic results $[12,13,15]$. These results were computed using the local part of the one loop effective action derived in $[73,78]$ for which one could use Wald's formula [1] or equivalently the classical entropy function formalism [5]. This local effective action, computed in type IIB string theory on $K 3 \times T^{2}$ and its various orbifolds, included contribution from massive string states carrying winding and momentum along the cycles of $T^{2}$, but the contribution due to the massless modes had to be removed by hand so as to avoid infrared divergent results. In contrast our analysis in this paper computes part of the contribution from the massless sector. Thus this contribution must be added to the result of the previous analysis.

Logarithmic corrections to the (extremal) black hole entropy have been analyzed before from different points of view $[49,70-72,77,79-86]$. The previous approaches can be divided into two broad classes, - microscopic and macroscopic. In the microscopic analysis the logarithmic corrections are computed by using specific microscopic description of the theory, while in the macroscopic approach the logartithmic corrections are computed from the analysis of fluctuating quantum fields in a black hole background. The macroscopic approaches can also be divided into two categories. In one category, that involves entropy of BTZ black holes, one first analyzes the gravity path integral in asymptotically $A d S_{3}$ 
spaces to compute the partition function, and then computes the entropy by taking a laplace transform of the partition function. In this approach the logarithmic terms arise in the process of taking the Laplace transform. In the second category one computes the entropy directly by analyzing the quantum fluctuations of various fields in the black hole geometry. The analysis of this paper clearly falls in the last category.

To be more specific, we shall now compare our method to [77] which is closest in spirit. In [77] logarithmic corrections to the entropy of extremal black hole was calculated by relating it to the partition function of the theory in the near horizon geometry with a conical defect. This requires computing the heat kernel of various fields in a background with conical defect. However while attempting to make this into a general prescription for computing black hole entropy in string theory, one runs into the problem that string theory may not make sense in backgrounds with arbitrary conical defects other than those obtained by taking orbifolds of smooth space-time. Our approach also requires computing the heat kernel of various fields, but directly in the near horizon geometry without a conical defect. As a result it is completely well defined once we adopt the infrared subtraction procedure described in [50]. Nevertheless the results of our approach agree with those of [77] for cases where both methods have been applied e.g. for a massles scalar field. The main advantage of our approach is that we begin with a general prescription for computing the entropy of an extremal black hole based on $A d S_{2} / C F T_{1}$ correspondence, and then evaluate it using various approximations. This allows us to carry out a systematic comparison between the macroscopic and microscopic entropies. So far (including the results of this paper) this comparison includes classical Wald entropy and some one loop and non-perturbative results.

The rest of the paper is organised as follows. In section 2 we show how quantum entropy function can be used to calculate logarithmic correction to the entropy of an extremal black hole due to a single massless scalar field coupled to the background metric by minimal coupling. This requires computing the eigenvalues and eigenfunctions of the scalar Laplacian in the near horizon geometry, and the heat kernel constructed from these data. We also study the effect of introducing a mass term for the scalar, and show that massive stringy states do not give any logarithmic correction to the entropy of an extremal black hole. In section 3 we generalize the analysis to include contribution from massless vector, $p$ form and spinor fields coupled to the background metric via minimal coupling. In section 4 we focus on the near horizon geometry of quarter BPS black holes in $\mathcal{N}=4$ supersymmetric string theories. By expanding the supergravity action in this near horizon background we find the complete quadratic action involving the various fluctuating fields in the matter and gravity multiplet. This action contains the minimal coupling of various fields to the background metric, but also contains additional terms including mixing between fields of different spin. We find however that at the quadratic order there is no mixing between the fluctuations in the matter and gravity multiplet fields, and hence we can separately analyze the one loop contribution from the two sets of fields. In section 5 we find the eigenvalues of the kinetic operator in the matter multiplet (which in general contains a mixing between the scalar and the vector fields, and also between different components of the spin half field) and use this to compute the one loop contribution to the quantum entropy function. We find that while individual fields give logarithmic contribution to 
the entropy, the total logarithmic contribution from each vector multiplet vanishes, in agreement with the microscopic results. We end in section 6 by summarizing the results and speculating on the possible application of the pure spinor formalism for a one loop computation of the quantum entropy function in full string theory. In appendix A we analyze the contribution from integration over the zero modes which were left out from the functional integral in the analysis of section 5 and show that they do not give any additional logarithmic correction to the black hole entropy.

\section{Logarithmic correction to the black hole entropy due to a single scalar field}

Suppose we have an extremal black hole with near horizon geometry $A d S_{2} \times S^{2}$, with equal size $a$ of $A d S_{2}$ and $S^{2}$. Then the Euclidean near horizon metric takes the form

$$
a^{2}\left(d \eta^{2}+\sinh ^{2} \eta d \theta^{2}\right)+a^{2}\left(d \psi^{2}+\sin ^{2} \psi d \phi^{2}\right),
$$

where $\theta$ and $\phi$ are periodic coordinates with period $2 \pi$. We choose the sign convention for the euclidean action $\mathcal{S}$ such that the weight factor inserted into the path integral is given by $e^{\mathcal{S}}$. Let $\Delta \mathcal{L}_{\text {eff }}$ denote the one loop correction to the four dimensional effective lagrangian density evaluated in the background geometry (2.1). Then the one loop correction to the action is given by

$$
\Delta \mathcal{S}=\int \sqrt{\operatorname{det} g} d \eta d \theta d \psi d \phi \Delta \mathcal{L}_{\mathrm{eff}}=8 \pi^{2} a^{4}\left(\cosh \eta_{0}-1\right) \Delta \mathcal{L}_{\mathrm{eff}}
$$

where $\eta_{0}$ is an infrared cut-off. The term proportional to $\cosh \eta_{0}$ has the interpretation of $-\beta \Delta E_{0}+\mathcal{O}\left(\beta^{-1}\right)$ where $\beta=2 \pi a \sinh \eta_{0}$ is the inverse temperature given by the length of the boundary of $A d S_{2}$ parametrized by $\theta$ and $\Delta E_{0}$ is the shift in the ground state energy $[10,50]$. The rest of the contribution can be interpreted as the one loop correction to the black hole entropy $[10,50]$ and takes the form

$$
\Delta S_{B H}=-8 \pi^{2} a^{4} \Delta \mathcal{L}_{\text {eff }} .
$$

We shall now describe the general procedure for calculating $\Delta \mathcal{L}_{\text {eff }}$.

Let us assume that the theory contains a massless scalar field. If we denote the eigenvalues of the scalar laplacian by $\left\{-\kappa_{n}\right\}$ and the corresponding normalized eigenfunctions by $f_{n}(x)$ then the heat kernel $K^{s}\left(x, x^{\prime} ; s\right)$ of the scalar Laplacian is defined as (see $[87,88]$ and references therein)

$$
K^{s}\left(x, x^{\prime} ; s\right)=\sum_{n} e^{-\kappa_{n} s} f_{n}(x) f_{n}\left(x^{\prime}\right) .
$$

The superscript $s$ on $K$ reflects that the laplacian acts on the scalar fields. In (2.4) we have assumed that we are working in a basis in which the eigenfunctions are real; if this is not the case then we need to take the complex conjugate of $f_{n}\left(x^{\prime}\right) . K^{s}\left(x, x^{\prime} ; s\right)$ satisfies the equation

$$
\left(\partial_{s}-\square_{x}\right) K^{s}\left(x, x^{\prime} ; s\right)=0 ; \quad K^{s}\left(x, x^{\prime} ; s=0\right)=\delta^{(4)}\left(x-x^{\prime}\right),
$$


$\square_{x}$ being the Laplacian on $A d S_{2} \times S^{2}$. The contribution of this scalar field to the one loop effective action can now be expressed as

$$
\Delta \mathcal{S}=-\frac{1}{2} \sum_{n} \ln \kappa_{n}=\frac{1}{2} \int_{\epsilon}^{\infty} \frac{d s}{s} \sum_{n} e^{-\kappa_{n} s}=\frac{1}{2} \int_{\epsilon}^{\infty} \frac{d s}{s} \int d^{4} x \sqrt{\operatorname{det} g} K^{s}(x, x ; s),
$$

where $g_{\mu \nu}$ is the $A d S_{2} \times S^{2}$ metric and $\epsilon$ is an ultraviolet cut-off. Comparing this with (2.2) we see that ${ }^{6}$

$$
\Delta \mathcal{L}_{\mathrm{eff}}=\frac{1}{2} \int_{\epsilon}^{\infty} \frac{d s}{s} K^{s}(0 ; s)
$$

where $K^{s}(0 ; s) \equiv K^{s}(x, x ; s)$. Note that using the fact that $A d S_{2}$ and $S^{2}$ are homogeneous spaces we have dropped the dependence on $x$ from $K^{s}(x, x ; s)$.

Now it follows from (2.4) and the fact that $\square_{A d S_{2} \times S^{2}}=\square_{A d S_{2}}+\square_{S^{2}}$ that the heat kernel of a massless scalar field on $A d S_{2} \times S^{2}$ is given by the product of the heat kernels on $A d S_{2}$ and $S^{2}$, and in the $x^{\prime} \rightarrow x$ limit takes the form [92]

$$
K^{s}(0 ; s)=K_{A d S_{2}}^{s}(0 ; s) K_{S^{2}}^{s}(0 ; s) .
$$

$K_{S^{2}}^{s}$ and $K_{A d S_{2}}^{s}$ in turn can be calculated using (2.4) if we know the eigenfunctions and the eigenvalues of the Laplace operator on these respective spaces. Fortunately these have been studied extensively [92-95]. On $S^{2}$ the normalized eigenfunctions of $-\square$ are just the usual spherical harmonics $Y_{l m}(\psi, \phi) / a$ with eigenvalues $l(l+1) / a^{2}$. Since $Y_{l m}$ vanishes at $\psi=0$ for $m \neq 0$, and $Y_{l 0}=\sqrt{2 l+1} / \sqrt{4 \pi}$ at $\psi=0$ we have

$$
K_{S^{2}}^{s}(0 ; s)=\frac{1}{4 \pi a^{2}} \sum_{l} e^{-s l(l+1) / a^{2}}(2 l+1) .
$$

On the other hand on $A d S_{2}$ the $\delta$-function normalized eigenfunctions of $-\square$ are given by [93]

$$
\begin{aligned}
& f_{\lambda, k}(\eta, \theta)= \frac{1}{\sqrt{2 \pi a^{2}}} \frac{1}{2^{|k|}(|k|) !}\left|\frac{\Gamma\left(i \lambda+\frac{1}{2}+|k|\right)}{\Gamma(i \lambda)}\right| e^{i k \theta} \sinh ^{|k|} \eta \\
& F\left(i \lambda+\frac{1}{2}+|k|,-i \lambda+\frac{1}{2}+|k| ;|k|+1 ;-\sinh ^{2} \frac{\eta}{2}\right), \\
& k \in \mathbb{Z}, \quad 0<\lambda<\infty,
\end{aligned}
$$

with eigenvalue $\left(\frac{1}{4}+\lambda^{2}\right) / a^{2}$. Here $F$ denotes hypergeometric function. Since the eigenfunction described in (2.10) vanishes at $\eta=0$ for $k \neq 0$, only the $k=0$ states will contribute to $K_{A d S_{2}}^{s}(0 ; s)$. At $\eta=0$ the $k=0$ state has the value $\sqrt{\lambda \tanh (\pi \lambda)} / \sqrt{2 \pi a^{2}}$. Thus $(2.4)$ gives

$$
K_{A d S_{2}}^{s}(0 ; s)=\frac{1}{2 \pi a^{2}} \int_{0}^{\infty} d \lambda \lambda \tanh (\pi \lambda) \exp \left[-s\left(\lambda^{2}+\frac{1}{4}\right) / a^{2}\right]
$$

\footnotetext{
${ }^{6}$ There are various other methods for evaluating functional determinants in non-trivial space-time backgrounds, see e.g. [89-91]. However the form of the result given in (2.7) is closest to the form in which we expect to obtain the answer in string theory, with the integration over $s$ replaced by integration over the modular parameter of a torus and $K^{s}(0 ; s)$ replaced by the torus partition function of the first quantized string.
} 
Combining (2.9) and (2.11) we get the heat kernel of a scalar field on $A d S_{2} \times S^{2}$ :

$$
K^{s}(0 ; s)=\frac{1}{8 \pi^{2} a^{4}} \sum_{\ell=0}^{\infty}(2 l+1) \int_{0}^{\infty} d \lambda \lambda \tanh (\pi \lambda) \exp \left[-\bar{s} \lambda^{2}-\bar{s}\left(l+\frac{1}{2}\right)^{2}\right]
$$

where

$$
\bar{s}=s / a^{2} .
$$

The associated eigenstates of the laplacian operator on $A d S_{2} \times S^{2}$ are obtained by taking the products of the spherical harmonics and the function $f_{\lambda, k}$ given in (2.10) and satisfy

$$
\square f_{\lambda, k}(\eta, \theta) Y_{l m}(\psi, \phi)=-\frac{1}{a^{2}}\left\{l(l+1)+\lambda^{2}+\frac{1}{4}\right\} f_{\lambda, k}(\eta, \theta) Y_{l m}(\psi, \phi) .
$$

We can in principle evaluate the full one loop effective action due to massless fields using (2.7) and (2.12), but our goal is to extract the piece proportional to $\ln a$ for large a. Such contributions come from the region of integration $1 \ll s \ll a^{2}$ or equivalently $a^{-2} \ll \bar{s} \ll 1$. Thus we need to study the behaviour of $(2.9),(2.11)$ for small $\bar{s}$. Since both $K_{S^{2}}^{s}(0 ; s)$ and $K_{A d S_{2}}^{s}(0 ; s)$ diverge at $\bar{s}=0$, we cannot simply expand the summand /integrand in (2.9), (2.11) in a power series expansion in $\bar{s}$, - we must first isolate the divergent part and evaluate it exactly. Let us begin with the expression for $K_{A d S_{2}}^{s}(0 ; s)$ given in (2.11). We first express $\tanh (\pi \lambda)$ as $1-2 e^{-2 \pi \lambda} /\left(1+e^{-2 \pi \lambda}\right)=1+2 \sum_{k=1}^{\infty}(-1)^{k} e^{-2 k \pi \lambda}$, and divide the integral into two parts: the first part containing the 1 term from the expansion of $\tanh (\pi \lambda)$ and the second part containing the rest of the terms. The first integral can be evaluated in closed form. In the second integral we expand $e^{-\bar{s} \lambda^{2}}$ in a power series expansion in $\bar{s}$ and perform the integral over $\lambda$. This leads to the following expression for $K_{A d S_{2}}^{s}:$

$$
\begin{aligned}
K_{A d S_{2}}^{s}(0 ; s) & =\frac{1}{4 \pi a^{2} \bar{s}} e^{-\bar{s} / 4}\left[1+\sum_{n=0}^{\infty} \frac{(-1)^{n}}{n !}(2 n+1) ! \frac{\bar{s}^{n+1}}{\pi^{2 n+2}} \frac{1}{2^{2 n}}\left(2^{-2 n-1}-1\right) \zeta(2 n+2)\right] \\
& =\frac{1}{4 \pi a^{2} \bar{s}} e^{-\bar{s} / 4}\left(1-\frac{1}{12} \bar{s}+\frac{7}{480} \bar{s}^{2}+\mathcal{O}\left(\bar{s}^{3}\right)\right)
\end{aligned}
$$

In order to find the small $s$ expansion of $K_{S^{2}}^{s}$, we first express (2.9) as

$$
\frac{1}{4 \pi i a^{2}} e^{\bar{s} / 4} \oint d \lambda \lambda \tan (\pi \lambda) e^{-\bar{s} \lambda^{2}}
$$

where $\oint$ denotes integration along a contour that travels from from $\infty$ to 0 staying below the real axis and returns to $\infty$ staying above the real axis. By deforming the integration contour to a pair of straight lines through the origin — one at an angle $\kappa$ below the positive real axis and the other at an angle $\kappa$ above the positive real axis - we can express this as

$$
\frac{1}{2 \pi a^{2}} e^{\bar{s} / 4} \operatorname{Im} \int_{0}^{e^{i \kappa} \times \infty} \lambda d \lambda \tan (\pi \lambda) e^{-\bar{s} \lambda^{2}}, \quad 0<\kappa \ll 1
$$


This integral can now be expressed in the same way as in the case of $K_{A d S_{2}}^{s}$, and we get

$$
\begin{aligned}
K_{S^{2}}^{s}(0 ; s) & =\frac{1}{4 \pi a^{2} \bar{s}} e^{\bar{s} / 4}\left[1-\sum_{n=0}^{\infty} \frac{1}{n !}(2 n+1) ! \frac{\bar{s}^{n+1}}{\pi^{2 n+2}} \frac{1}{2^{2 n}}\left(2^{-2 n-1}-1\right) \zeta(2 n+2)\right] \\
& =\frac{1}{4 \pi a^{2} \bar{s}} e^{\bar{s} / 4}\left(1+\frac{1}{12} \bar{s}+\frac{7}{480} \bar{s}^{2}+\mathcal{O}\left(\bar{s}^{3}\right)\right)
\end{aligned}
$$

Substituting (2.15) and (2.18) into (2.8) we get

$$
K^{s}(0 ; s)=\frac{1}{16 \pi^{2} a^{4} \bar{s}^{2}}\left(1+\frac{1}{45} \bar{s}^{2}+\mathcal{O}\left(s^{4}\right)\right) .
$$

eq. (2.7) now gives

$$
\Delta \mathcal{L}_{\mathrm{eff}}=\frac{1}{32 \pi^{2} a^{4}} \int_{\epsilon / a^{2}}^{\infty} \frac{d \bar{s}}{\bar{s}^{3}}\left(1+\frac{1}{45} \bar{s}^{2}+\mathcal{O}\left(\bar{s}^{4}\right)\right)
$$

This integral has a quadratically divergent piece proportional to $1 / \epsilon^{2}$. This can be thought of as a renormalization of the cosmological constant and will cancel against contribution from other fields in a supersymmetric theory in which the cosmological constant is not renormalized. Even otherwise in string theory there is a physical cut-off set by the string scale. ${ }^{7}$ Our main interest is in the logarithmically divergent piece which comes from the order $\bar{s}^{2}$ term inside the parentheses. This is given by

$$
\frac{1}{1440 \pi^{2} a^{4}} \ln \left(a^{2} / \epsilon\right),
$$

and, according to (2.3) gives a contribution to the entropy

$$
\Delta S_{B H}=-\frac{1}{180} \ln \left(a^{2} / \epsilon\right) .
$$

This agrees with the earlier result of $[77,96]$. In this earlier approach one computed the black hole entropy by relating it to the partition function of the theory in an eucldean space-time with a conical defect [97]. This required computing the scalar heat kernel on a space-time with conical defect. Besides being computationally more difficult, this method also suffered from the intrinsic problem that string theory on a space-time with conical defect may not be well defined. In contrast the quantum entropy function approach only requires us to compute the partition function of string theory on the near horizon $A d S_{2} \times S^{2}$ geometry. Since this is a smooth geometry, and solves the classical equations of motion of string theory, the partition function of the theory in this space should be well defined, leading to an unambiguous prescription for computing the black hole entropy.

Note that the term in $\mathcal{L}_{\text {eff }}$ proportional to $\ln a^{2}$ comes from the $s$ independent part of $K^{s}(0 ; s)$ in an expansion in $s$. This can also be calculated using the general formula

\footnotetext{
${ }^{7}$ Typically in a string theory there are multiple scales e.g. string scale, Planck scale, scale set by the mass of the D-branes etc. We shall consider near horizon background where the string coupling constant as well as all the other parameters describing the shape, size and the various background fields along the six compact directions are of order unity. In this case all these length scales will be of the same order.
} 
derived in $[87,88,98-101]$ which relates the coefficients appearing in the small $s$ expansion of $K(0 ; s)$ to local quantities computed in the background geometry. In $A d S_{2} \times S^{2}$, where the Weyl tensor as well as the curvature scalar vanishes, the formula for the constant part of $K^{s}(0 ; s)$, denoted as $a_{4}^{s}(0 ; s)$, takes the form

$$
a_{4}^{s}(0 ; s)=\frac{1}{180(4 \pi)^{2}} R_{\mu \nu} R^{\mu \nu},
$$

where $R_{\mu \nu}$ is the Ricci scalar. Evaluating it on the background (2.1) we find that $R_{\mu \nu} R^{\mu \nu}=$ $4 a^{-4}$, and hence

$$
a_{4}^{s}(0 ; s)=\frac{1}{16 \pi^{2} a^{4}} \frac{1}{45} .
$$

This is in precise agreement with the coefficient of the $s$ independent term in (2.19). We shall see however that evaluating the heat kernel explicitly by summing over eigenfunctions gives us valuable insight that will be useful for our analysis when we try to extend the results to include the contribution from higher spin fields, and the effect of background electric and magnetic fluxes on $A d S_{2} \times S^{2}$. In particular in order that the integrals of the form appearing in (2.7) are well defined, we need to subtract from the integrand $K(0, s)$ its value at $s \rightarrow \infty$, - this corresponds to removing the zero eigenvalues of the kinetic operator from the definition of the determinant. Thus we need to know the $s \rightarrow \infty$ limit of the heat kernel besides its small $s$ expansion.

Let us now discuss the effect of switching on a mass term for the scalar. The effect of this is to insert a factor of $e^{-m^{2} s}=e^{-m^{2} a^{2} \bar{s}}$ into the integral in (2.20). This gives

$$
\Delta \mathcal{L}_{\text {eff }}=\frac{1}{32 \pi^{2} a^{4}} \int_{\epsilon / a^{2}}^{\infty} \frac{d \bar{s}}{\bar{s}^{3}}\left(1+\frac{1}{45} \bar{s}^{2}+\mathcal{O}\left(\bar{s}^{4}\right)\right) e^{-m^{2} a^{2} \bar{s}}
$$

We shall now consider two different situations. First suppose $m^{2}$ is of order unity, r.e. of the order of the string scale. In that case the exponential factor in (2.25) effectively restricts the integration over $\bar{s}$ in the region $\bar{s}_{\sim}^{<} 1 / a^{2}$. As a result $\Delta \mathcal{L}_{\text {eff }}$ will not contain any piece proportional to $\ln a^{2}$. On the other hand if $m^{2}=c / a^{2}$ where $c$ is a constant of order unity, then in the region $\bar{s} \ll 1$ the term in the exponent is small and we can expand the exponential in a Taylor series expansion. This gives

$$
\Delta \mathcal{L}_{\mathrm{eff}}=\frac{1}{32 \pi^{2} a^{4}} \int_{\epsilon / a^{2}}^{\infty} \frac{d \bar{s}}{\bar{s}^{3}}\left(1-c \bar{s}+\frac{1}{2} c^{2} \bar{s}^{2}+\frac{1}{45} \bar{s}^{2}+\mathcal{O}\left(\bar{s}^{4}\right)\right) .
$$

This has a term proportional to $\ln a^{2}$ of the form

$$
\frac{1}{8 \pi^{2} a^{4}}\left(\frac{1}{180}+\frac{c^{2}}{8}\right) \ln \left(a^{2}\right)
$$

and, according to (2.3) gives a contribution to the entropy

$$
\Delta S_{B H}=-\left(\frac{1}{180}+\frac{c^{2}}{8}\right) \ln \left(a^{2}\right) .
$$

This shows that a massive scalar whose mass is of the order of string scale does not give any contribution to the entropy proportional to $\ln a^{2}$. On the other hand a massive scalar 
whose mass is inversely proportional to $a$ will contribute terms proportional to $\ln a$ to the entropy, and furthermore the actual contribution will depend on the mass of the scalar.

In the examples we shall analyze, the presence of the background flux generates an effective potential of order $a^{-2}$ for background scalars via the coupling between scalars and vector fields in the supergravity action. Thus we must take into account such corrections in our analysis. In contrast the massive string modes have mass of the order of the string scale and we can ignore their contribution while computing logarithmic correction to the black hole entropy. Finally one might also worry about the effect of higher derivative corrections to the effective action on the potential for the scalars. Such corrections are of order $a^{-4}$ or higher powers of $a^{-1}$, and do not affect the logarithmic correction to the entropy since correction to the exponent $m^{2} a^{2} \bar{s}$ remains small throughout the relevant region of integration.

So far we have discussed the effect of one loop corrections. What about higher loop contributions? As discussed in footnote 7, we have assumed that all the moduli parameters including the string coupling constant are of order unity at the horizon; hence the higher loop contributions could be of the same order as the one loop contribution. While we cannot make any definite prediction about these higher loop corrections in general, in the special case of supersymmetric black holes we shall be considering, we can argue as follows that the higher loop contributions can be ignored. For definiteness we shall consider a situation where all the charges carried by the black hole are Ramond-Ramond (RR) charges. In this case the scaling argument of [102] tells us that as we scale all the charges by some common scale $\Lambda$, the dilaton $\Phi$ at the horizon scales as $e^{-2 \Phi} \sim \Lambda^{2}$, and all the other NSNS background fields, including the string metric, remains fixed. As a result for large $\Lambda$ the four dimensional canonical metric $g_{\mu \nu}$, related to the string metrc $G_{\mu \nu}$ via $g_{\mu \nu}=e^{-2 \Phi} G_{\mu \nu}$, scales as $\Lambda^{2}$ and $e^{-2 \Phi}$ scales as $\Lambda^{2}$. This would seem to contradict our assumption of footnote 7 that all the moduli are of order one at the horizon. This is resolved by noting that at least in the classical supergravity approximation, the value of the dilaton at the horizon can be changed keeping the four dimensional canonical metric fixed. In the language of $\mathcal{N}=2$ supersymmetric theories this is a consequence of the fact that the four dimensional dilaton belongs to the hypermultiplet and hence the vector multiplet fields do not generate any potential for the dilaton. We shall assume that this flat direction, labelled by the value of the dilaton, is not lifted even in the full quantum theory. Thus we can evaluate the entropy at any value of the dilaton, in particular either for $e^{-2 \Phi} \sim \Lambda^{2}$ as given by the scaling argument or for $e^{-2 \Phi} \sim 1$. In the first case the $k$ loop contribution to the entropy will go as $e^{2 \Phi(k-1)} \sim \Lambda^{2-2 k}$. In the second case we have $e^{2 \Phi} \sim 1$ and $G_{\mu \nu}=e^{2 \Phi} g_{\mu \nu} \sim \Lambda^{2}$. Thus any term that has $2 k+2$ derivatives will give a contribution of order $\Lambda^{2-2 k}$ irrespective of the order of the perturbation theory in which it is generated. Requiring that both be correct leads to the conclusion that at $k$ loop order only the terms with $2 k+2$ derivatives will contribute to the entropy, giving a contribution of order $\Lambda^{2-2 k}$. Thus the logarithmic corrections can arise only from one loop terms in the effective action, the higher loop corrections being suppressed by inverse powers of $\Lambda$, 1.e. inverse powers of the charges. 


\section{Heat kernels of vector, $p$-form and fermion fields}

The matter multiplet of an $\mathcal{N}=4$ supersymmetric theory in $(3+1)$ dimensions contains a vector, four Majorana fermions and six scalars. Thus in order to compute the logarithmic correction to the entropy due to a matter multiplet we need to extend the results of the previous section to include the heat kernels of vector and fermion fields. In this section we shall compute the heat kernels of these fields by regarding them as free fields in $A d S_{2} \times S^{2}$ background. Although the analysis is straightforward using the results of [92-95, 103], we shall go through it carefully, since, as we shall see in the next two sections, these results need to be further corrected due to mixing between scalar, vector and tensor fields in the black hole near horizon geometry.

\subsection{Vector fields}

In general the contribution from a field of given spin requires evaluation of the functional integral after suitable gauge fixing. We shall use a Feynman type gauge and compute the net contribution from a given field as the sum of the contribution from the original field as well as the various ghosts which appear during gauge fixing. Let us first consider the case of a U(1) gauge field with euclidean action

$$
\mathcal{S}_{A}=-\frac{1}{4} \int d^{4} x \sqrt{\operatorname{det} g} F_{\mu \nu} F^{\mu \nu},
$$

where $F_{\mu \nu} \equiv \partial_{\mu} A_{\nu}-\partial_{\nu} A_{\mu}$ is the gauge field strength. Adding a gauge fixing term

$$
S_{g f}=-\frac{1}{2} \int d^{4} x \sqrt{\operatorname{det} g}\left(D_{\mu} A^{\mu}\right)^{2},
$$

we can express the action as

$$
\mathcal{S}_{A}+\mathcal{S}_{g f}=-\frac{1}{2} \int d^{4} x \sqrt{\operatorname{det} g} A_{\mu}(\Delta A)^{\mu},
$$

where

$$
(\Delta A)_{\mu} \equiv-\square A_{\mu}+R_{\mu \nu} A^{\nu}, \quad \square A_{\mu} \equiv g^{\rho \sigma} D_{\rho} D_{\sigma} A_{\mu} .
$$

We shall denote by $d$ the exterior derivative operator and by $\delta$ the operator $-* d *$ where $*$ denotes Hodge dual operation. Then $\Delta$ may be expressed as

$$
\Delta \equiv(d \delta+\delta d) \text {. }
$$

We shall use (3.5) as the definition of $\Delta$ acting on any $p$-form field.

Since the eigenfunctions of $\Delta$ are four component vectors, the vector heat kernel is a $4 \times 4$ matrix. We shall denote by $K^{v}\left(x, x^{\prime} ; s\right)$ the trace of this matrix. Quantization of gauge fields also requires us to introduce two anticommuting scalar ghosts whose kinetic operator is given by the standard laplacian $-\square=\delta d$ in the harmonic gauge. Thus the net one loop contribution of the vector field to $\mathcal{L}_{\text {eff }}$ will be given by

$$
\frac{1}{2} \int_{\epsilon}^{\infty} \frac{d s}{s} \sqrt{\operatorname{det} g} \lim _{x^{\prime} \rightarrow x}\left[K^{v}\left(x, x^{\prime} ; s\right)-2 K^{s}\left(x, x^{\prime} ; s\right)\right],
$$

where the $-2 K^{s}$ term reflects the contribution due to the ghosts.

A vector in $A d S_{2} \times S^{2}$ decomposes into a (vector, scalar) plus a (scalar, vector), with the first and the second factors representing tensorial properties in $A d S_{2}$ and $S^{2}$ 
respectively. Furthermore, on any of these components the action of the kinetic operator can be expressed as $\Delta_{A d S_{2}}+\Delta_{S^{2}}$, with $\Delta$ as defined in (3.5). Thus we can construct the eigenfunctions of $\Delta$ by taking the product of appropriate eigenfunctions of $\Delta_{A d S_{2}}$ and $\Delta_{S^{2}}$, and the corresponding eigenvalue of $\Delta$ on $A d S_{2} \times S^{2}$ will be given by the sum of the eigenvalues of $\Delta_{A d S_{2}}$ and $\Delta_{S^{2}}$. This gives ${ }^{8}$

$$
K^{v}(0 ; s)=K_{A d S_{2}}^{v}(0, s) K_{S^{2}}^{s}(0 ; s)+K_{A d S_{2}}^{s}(0, s) K_{S^{2}}^{v}(0 ; s) .
$$

Thus we need to compute $K_{A d S_{2}}^{v}(0, s)$ and $K_{S^{2}}^{v}(0 ; s)$.

Now suppose that we have a scalar field $\Phi$ on $A d S_{2}$ or $S^{2}$ satisfying

$$
\Delta \Phi \equiv \delta d \Phi \equiv-\square \Phi=\kappa \Phi
$$

Then we can construct two configurations for the gauge field $A$ with the same eigenvalue $\kappa$ of $\Delta$ and the same normalization as $\Phi$ as follows:

$$
A^{(1)}=\kappa^{-1 / 2} d \Phi, \quad A^{(2)}=\kappa^{-1 / 2} * d \Phi .
$$

Furthermore locally every vector field in two dimensions can be decomposed as $d \Phi_{1}+$ $* d \Phi_{2}$. Thus for every scalar eigenfunction $\Phi$ of the operator $\delta d$ we have a pair of vector eigenfunctions of $(d \delta+\delta d)$ with the same eigenvalue. The contribution from any of these two eigenfunctions to the vector heat kernel $K^{v}(x, x ; s)$ is given by $\kappa^{-1} e^{-\kappa s} g^{\mu \nu} \partial_{\mu} \Phi(x) \partial_{\nu} \Phi(x)$. Now since $K^{v}(x, x ; s)$ is independent of $x$ after summing over the contribution from all the states, we could compute it by taking the volume average of each term. Taking a volume average allows us to integrate by parts and gives the same result as the volume average of $\kappa^{-1} e^{-\kappa s} \Phi(x) \delta d \Phi(x)=e^{-\kappa s} \Phi(x)^{2}$. This is the same as the contribution from $\Phi(x)$ to the scalar heat kernel. ${ }^{9}$ Thus we conclude that leaving aside global issues, the heat kernel for a vector field on $A d S_{2}$ or $S^{2}$ should be given by twice that of the scalar.

There are however some corrections to this both on $S^{2}$ and $A d S_{2}$ due to global issues. On $S^{2}$, the constant mode of the scalar is an eigenfunction of $\square_{S^{2}}$ with eigenvalue 0 . However these modes do not generate any non-trivial gauge field configuration via (3.9). Hence their contribution to $K_{S^{2}}^{s}$ should be removed while computing $K_{S^{2}}^{v}$. Since the zero mode gives a contribution of $1 /\left(4 \pi a^{2}\right)$ to $K_{S^{2}}^{s}(0 ; s)$, this gives

$$
K_{S^{2}}^{v}(0, s)=2 K_{S^{2}}^{s}(0, s)-\frac{1}{2 \pi a^{2}} .
$$

On the other hand on $A d S_{2}$ the constant mode of the scalar is not normalizable, and hence $K_{A d S_{2}}^{s}$ does not include any contribution from the constant mode. Thus we do not need to make any subtraction from $K_{A d S_{2}}^{s}$ in computing $K_{A d S_{2}}^{v}$. However it turns out that in

\footnotetext{
${ }^{8}$ The main ingradient that allows us to express the heat kernel on $A d S_{2} \times S^{2}$ in terms of heat kernels on $A d S_{2}$ and $S^{2}$ is that the kinetic operator on $A d S_{2} \times S^{2}$ can be expressed as a sum of the kinetic operators in $S^{2}$ and $A d S_{2}$. This will continue to hold for the other fields as well, but the choice of harmonic gauge is essential for this.

${ }^{9}$ This can also be verified using the explicit form of the scalar eigenmodes given in section 2 and noting that the non-vanishing contribution now comes from eigenmodes with $Y_{l, \pm 1}$ on $S^{2}$ and $f_{\lambda, \pm 1}$ on $A d S_{2}$.
} 
this case there is a set of square integrable eigenvectors of $\Delta$ with zero eigenvalue, given by $[93]:{ }^{10}$

$$
A=d \Phi, \quad \Phi=\frac{1}{\sqrt{2 \pi|\ell|}}\left[\frac{\sinh \eta}{1+\cosh \eta}\right]^{|\ell|} e^{i \ell \theta}, \quad \ell= \pm 1, \pm 2, \pm 3, \cdots .
$$

These are not included in (3.9) since the $\Phi$ given in (3.11) is not normalizable. These give additional contribution to $K_{A d S_{2}}^{v}(0 ; s)$. In fact since for $|\ell|>1$ the gauge field vanishes at $\eta=0$, only the $\ell= \pm 1$ terms contribute to $K_{A d S_{2}}^{v}(0 ; s)$. This gives

$$
K_{A d S_{2}}^{v}(0, s)=2 K_{A d S_{2}}^{s}(0, s)+\frac{1}{2 \pi a^{2}} .
$$

We now proceed to compute the contribution to the vector heat kernel using these results. Using (3.7), (3.10), (3.12) and then (2.15), (2.18) we get

$$
\begin{aligned}
K^{v}(0 ; s) & =4 K_{A d S_{2}}^{s}(0 ; s) K_{S^{2}}^{s}(0 ; s)+\frac{1}{2 \pi a^{2}}\left(K_{S^{2}}^{s}(0 ; s)-K_{A d S_{2}}^{s}(0 ; s)\right) \\
& =\frac{1}{4 \pi^{2} a^{4} \bar{s}^{2}}\left(1+\frac{16}{45} \bar{s}^{2}+\mathcal{O}\left(\bar{s}^{4}\right)\right) .
\end{aligned}
$$

This is again consistent with the results of [100] which gives the coefficient of the $s$ independent part of $K^{v}$ to be $64(180)^{-1}(4 \pi)^{-2} R_{\mu \nu} R^{\mu \nu}$. Taking into account the contribution due to the ghosts via (3.6) we can now compute the total contribution to the effective action from the vector field.

There is however an additional subtlety we must take care of. The contribution to the vector heat kernel given in (3.13) includes contribution from the zero modes obtained by taking the product of (3.11) and the $l=0$ mode, i.e. the constant mode of the scalar on $S^{2}$. The integration over the zero modes of any field requires special treatment since these integrals are not Gaussian. Thus in evaluating the determinant of the kinetic operator for computing the one loop contribution to the effective action we must remove the contribution due to the zero modes [104]. This will require replacing $K^{v}(0 ; s)$ by

$$
\widehat{K}^{v}(0 ; s)=K_{A d S_{2}}^{v}(0, s) K_{S^{2}}^{s}(0 ; s)+K_{A d S_{2}}^{s}(0, s) K_{S^{2}}^{v}(0 ; s)-\frac{1}{8 \pi^{2} a^{4}} .
$$

More generally, removal of the zero modes from any heat kernel will require subtracting from $K(0 ; s)$ its value as $s \rightarrow \infty$ :

$$
\widehat{K}(0 ; s) \equiv K(0 ; s)-\lim _{t \rightarrow \infty} K(0, t) .
$$

We shall take this as the definition of the proper heat kernel that should be used in computing logrithmic correction to the entropy. This subtraction is in fact necessary to ensure that the integration over $s$ does not diverge at infinity. ${ }^{11}$ However instead of removing the zero mode contribution from the heat kernel of every field we shall find it more convenient

\footnotetext{
${ }^{10}$ Since $d \Phi$ is (anti-)self-dual in $A d S_{2}$, we do not get independent eigenfunctions from $* d \Phi$.

${ }^{11}$ In any case a constant term in $K(0 ; s)$ will not produce a factor of $\ln a^{2}$, - these arise from terms which remain constant in the range $1 \ll s \ll a^{2}$ and fall off for $s \gg a^{2}$.
} 
to remove the contribution at the end from the trace of the total heat kernel of all the fields. For this reason we shall continue to use the result (3.13) for the vector heat kernel.

Even though in evaluating the determinant of the kinetic operator we need to remove the contribution due to the zero modes, eventually we must carry out the integration over the zero modes of physical fields. We shall describe the analysis of the zero mode integrals in appendix A and show that the net effect of these integrals - and an additional contribution that will be described in the same appendix - cancel, leaving us with the prescription of working with the regularized heat kernel described in (3.15).

\section{$3.2 p$-form fields}

A matter multiplet in $\mathcal{N}=4$ supergravity theory contains six scalar fields. However often in string theory, some of the scalars appear in their dual form as 2-form fields. This happens for example if we consider type IIA string theory on $K 3 \times T^{2}$, - we get 2 -form fields from taking the components of the RR 3-form field with one leg on $T^{2}$ and also from the NSNS sector 2-form fields. All of these need to be dualized to scalars and they then form parts of the matter multiplets. However from the viewpoint of type IIA string theory we should really carry out the path integral by regarding them as 2-form fields. Similarly in the same theory the RR 3-form field with all its legs along the four dimensional Minkowski space must also be regarded as an integration variable in the path integral even though in four dimensions it does not have any physical degree of freedom. Thus in order that our results do not depend on which description of the theory we use, we must ensure that the 2 -form field and the scalar gives the same contribution to the one loop determinant and that the 3 -form field does not contribute to the one loop determinant. We shall now try to verify this explicitly. This analysis is important in view of the results of [105] that the dual descriptions do not always lead to the same result for the trace of the stress tensor, which in turn can be related to the $s$ independent term in the expansion of $K(0 ; s)$.

First we consider the 2-form field $B_{\mu \nu}$ with gauge invariant action

$$
S_{B}=-\frac{1}{12} \int d^{4} x \sqrt{\operatorname{det} g} H_{\mu \nu \rho} H^{\mu \nu \rho}, \quad H_{\mu \nu \rho}=\partial_{\mu} B_{\nu \rho}+\partial_{\nu} B_{\rho \mu}+\partial_{\rho} B_{\mu \nu} .
$$

Adding a harmonic gauge fixing term

$$
S_{g f}=-\frac{1}{2} \int d^{4} x \sqrt{\operatorname{det} g} g^{\mu \nu} D^{\rho} B_{\rho \mu} D^{\sigma} B_{\sigma \nu},
$$

we get a simple form of the total action

$$
S_{B}+S_{g f}=-\frac{1}{2} \int d^{4} x \sqrt{\operatorname{det} g} B_{\mu \nu}(\Delta B)^{\mu \nu}, \quad \Delta B \equiv(d \delta+\delta d) B .
$$

On $A d S_{2}$ and $S^{2}$, there is a one to one correspondence between the normalizable modes of the scalar and the normalizable modes of $B_{\mu \nu}$ via Hodge duality $B_{\mu \nu}=\Phi \varepsilon_{\mu \nu}$ where $\varepsilon$ is the solume form. As a result the heat kernels for $B_{\mu \nu}$ and scalars are identical in these two spaces:

$$
K_{S^{2}}^{b}(0 ; s)=K_{S^{2}}^{s}(0 ; s), \quad K_{A d S_{2}}^{b}(0 ; s)=K_{A d S_{2}}^{s}(0 ; s)
$$


Since the 2-form field on $A d S_{2} \times S^{2}$ can be decomposed as (vector, vector), (2-form, scalar) and (scalar, 2-form), and furthermore on any of these components the action of the kinetic operator is given by $\Delta_{A d S_{2}}+\Delta_{S^{2}}$, we can express the trace of the heat kernel of the 2-form field on $A d S_{2} \times S^{2}$ as

$$
\begin{aligned}
K^{b}(0 ; s) & =K_{A d S_{2}}^{v}(0 ; s) K_{S^{2}}^{v}(0 ; s)+2 K_{A d S_{2}}^{s}(0 ; s) K_{S^{2}}^{s}(0 ; s) \\
& =6 K_{A d S_{2}}^{s}(0 ; s) K_{S^{2}}^{s}(0 ; s)+\frac{1}{\pi a^{2}}\left(K_{S^{2}}^{s}(0 ; s)-K_{A d S_{2}}^{s}(0 ; s)\right)-\frac{1}{4 \pi^{2} a^{4}} \\
& =\frac{1}{16 \pi^{2} a^{4}}\left(\frac{6}{\bar{s}^{2}}-\frac{6}{5}+\mathcal{O}\left(\bar{s}^{2}\right)\right)
\end{aligned}
$$

where we have used (3.19) in the first step, (3.10), (3.12) in the second step and (2.15), (2.18) in the last step. This is consistent with the results of [100] which gives the coefficient of the $s$ independent part of $K^{b}$ to be $-54(180)^{-1}(4 \pi)^{-2} R_{\mu \nu} R^{\mu \nu}$.

Quantization of the 2-form field produces two anti-commuting vector ghosts and three commuting scalar ghosts [106-108]. Thus the net contribution to the one loop effective action is given by

$$
\begin{aligned}
\Delta \mathcal{L}_{\mathrm{eff}} & =\frac{1}{2} \int_{\epsilon}^{\infty} \frac{d s}{s}\left[K^{b}(0 ; s)-2 K^{v}(0 ; s)+3 K^{s}(0 ; s)\right] \\
& =\frac{1}{2} \int_{\epsilon}^{\infty} \frac{d s}{s}\left[K^{s}(0 ; s)-\frac{1}{4 \pi^{2} a^{4}}\right] .
\end{aligned}
$$

Comparing (2.7) and (3.21) we see that the contribution to the one loop effective action due to a scalar field differs from that of a 2-form field. This is a bit surprising since in four dimensions the scalar and 2-form fields are supposed to be equivalent. As already noted in $[105,109]$, this difference can be attributed to the contribution due to the zero modes, - we shall now verify this explicitly. Indeed the zero mode contribution to the heat kernel can be identified as the term obtained by taking the $s \rightarrow \infty$ limit of the heat kernel. Thus on the right hand side of (3.21) this is given by the $-1 / 4 \pi^{2} a^{4}$ term in the square bracket. As discussed before, in calculating the one loop determinant we must explicitly remove the contribution due to the zero modes. In this case we shall no longer have the $-1 / 4 \pi^{2} a^{4}$ term inside the integrand in (3.21) and the result for the effective action computed using the 2-form field would agree with that computed using the scalar.

We can carry out a similar analysis for a 3 -form field in the harmonic gauge. In this gauge the kinetic operator is again given by $\Delta=(d \delta+\delta d)$. Since the 3 -form field on $A d S_{2} \times S^{2}$ and a vector field can be related by Hodge duality, the relevent part of the heat kernel for the 3 -form is given by $K^{v}(0 ; s)$. On the other hand the quantization of the 3 -form requires 2 anti-commuting 2 -form ghosts, 3 commuting vector ghosts and 4 anti-commuting scalar ghosts [106-108]. Thus the net contribution to $\Delta \mathcal{L}_{\text {eff }}$ is

$$
\begin{aligned}
\Delta \mathcal{L}_{\text {eff }} & =\frac{1}{2} \int_{\epsilon}^{\infty} \frac{d s}{s}\left[K^{v}(0 ; s)-2 K^{b}(0 ; s)+3 K^{v}(0 ; s)-4 K^{s}(0 ; s)\right] \\
& =\frac{1}{4 \pi^{2} a^{4}} \int_{\epsilon}^{\infty} \frac{d s}{s} .
\end{aligned}
$$


This is contrary to our expectation that the contribution to the effective action from a 3 -form field should vanish since it is non-dynamical in four dimensions. We now note that since the total heat kernel represented by the term inside the square bracket is an $s$-independent constant, removing the zero mode contribution amounts to subtracting this constant. This makes the net contribution vanish, in agreement with the general expectation.

\subsection{Fermions}

Next we turn to the computation of the heat kernel of spinors [103]. Consider a Dirac spinor $^{12}$ on $A d S_{2} \times S^{2}$. It decomposes into a product of a Dirac spinor on $A d S_{2}$ and a Dirac spinor on $S^{2}$. We use the following conventions for the vierbeins and the gamma matrices

$$
\begin{gathered}
e^{0}=a \sinh \eta d \theta, \quad e^{1}=a d \eta, \quad e^{2}=a \sin \psi d \phi, \quad e^{3}=a d \psi, \\
\Gamma^{0}=-\sigma_{3} \otimes \tau_{2}, \quad \Gamma^{1}=\sigma_{3} \otimes \tau_{1}, \quad \Gamma^{2}=-\sigma_{2} \otimes I_{2}, \quad \Gamma^{3}=\sigma_{1} \otimes I_{2},
\end{gathered}
$$

where $\sigma_{i}$ and $\tau_{i}$ are two dimensional Pauli matrices acting on different spaces and $I_{2}$ is $2 \times 2$ identity matrix. In this convention the Dirac operator on $A d S_{2} \times S^{2}$ can be written as

$$
\not D_{A d S_{2} \times S^{2}}=\not D_{S^{2}}+\sigma_{3} \not D_{A d S_{2}}
$$

where

$$
\not D_{S^{2}}=a^{-1}\left[-\sigma^{2} \frac{1}{\sin \psi} \partial_{\phi}+\sigma^{1} \partial_{\psi}+\frac{1}{2} \sigma^{1} \cot \psi\right]
$$

and

$$
\not D_{A d S_{2}}=a^{-1}\left[-\tau^{2} \frac{1}{\sinh \eta} \partial_{\theta}+\tau^{1} \partial_{\eta}+\frac{1}{2} \tau^{1} \operatorname{coth} \eta\right] .
$$

First let us analyze the eigenstates of $\not D_{S^{2}}$. They are given by [103]

$$
\begin{aligned}
& \chi_{l, m}^{ \pm}=\frac{1}{\sqrt{4 \pi a^{2}}} \frac{\sqrt{(l-m) !(l+m+1) !}}{l !} e^{i\left(m+\frac{1}{2}\right) \phi}\left(\begin{array}{c}
i \sin ^{m+1} \frac{\psi}{2} \cos ^{m} \frac{\psi}{2} P_{l-m}^{(m+1, m)}(\cos \psi) \\
\pm \sin ^{m} \frac{\psi}{2} \cos ^{m+1} \frac{\psi}{2} P_{l-m}^{(m, m+1)}(\cos \psi)
\end{array}\right), \\
& \eta_{l, m}^{ \pm}=\frac{1}{\sqrt{4 \pi a^{2}}} \frac{\sqrt{(l-m) !(l+m+1) !}}{l !} e^{-i\left(m+\frac{1}{2}\right) \phi}\left(\begin{array}{c}
\sin ^{m} \frac{\psi}{2} \cos ^{m+1} \frac{\psi}{2} P_{l-m}^{(m, m+1)}(\cos \psi) \\
\pm i \sin ^{m+1} \frac{\psi}{2} \cos ^{m} \frac{\psi}{2} P_{l-m}^{(m+1, m)}(\cos \psi)
\end{array}\right), \\
& l, m \in \mathbb{Z}, \quad l \geq 0, \quad 0 \leq m \leq l,
\end{aligned}
$$

satisfying

$$
\not D_{S^{2}} \chi_{l, m}^{ \pm}= \pm i a^{-1}(l+1) \chi_{l, m}^{ \pm}, \quad \not D_{S^{2}} \eta_{l, m}^{ \pm}= \pm i a^{-1}(l+1) \eta_{l, m}^{ \pm}
$$

Here $P_{n}^{\alpha, \beta}(x)$ are the Jacobi Polynomials:

$$
P_{n}^{(\alpha, \beta)}(x)=\frac{(-1)^{n}}{2^{n} n !}(1-x)^{-\alpha}(1+x)^{-\beta} \frac{d^{n}}{d x^{n}}\left[(1-x)^{\alpha+n}(1+x)^{\beta+n}\right] .
$$

\footnotetext{
${ }^{12}$ Even if the spinors satisfy Majorana/Weyl condition, we shall compute their heat kernel by first computing the result for a Dirac spinor and then taking appropriate square roots.
} 
We shall denote by $K_{S^{2}}^{f}\left(x, x^{\prime} ; s\right)$ the trace over the spinor indices of the heat kernel of the Dirac fermion on $S^{2}$. The precise normalization of $K_{S^{2}}^{f}$ is chosen as follows. If $\not D_{S^{2}}$ has eigenfunction $f_{n}(x)$ with eigenvalue $i \lambda_{n}$, then we define

$$
K_{S^{2}}^{f}\left(x, x^{\prime} ; s\right)=-\sum_{n} e^{-s \lambda_{n}^{2}} f_{n}^{\dagger}\left(x^{\prime}\right) f_{n}(x) .
$$

The extra minus sign in the definition of $K_{S^{2}}^{f}$ has been included to account for the fact that for fermionic path integral we get a factor of the determinant instead of the inverse of the determinant. Two additional normalization factors cancel; the fact that $i \not D$ is the square root of $-\not D^{2}$ gives a factor of $1 / 2$, but since we are considering a Dirac fermion instead of a Majorana fermion we get a factor of 2 . The result for (3.31) in the $x \rightarrow x^{\prime}$ limit can be simplified by noting that for $\psi=0, \chi_{l, m}^{ \pm}, \eta_{l, m}^{ \pm}$vanishes unless $m=0$, and

$$
\left(\chi_{l, 0}^{ \pm}\right)^{\dagger} \chi_{l, 0}^{ \pm}=\left(\eta_{l, 0}^{ \pm}\right)^{\dagger} \eta_{l, 0}^{ \pm}=\frac{1}{4 \pi a^{2}}(l+1)
$$

Thus we get

$$
K_{S^{2}}^{f}(0 ; s)=-\frac{1}{2 \pi a^{2}} \sum_{l=0}^{\infty}(2 l+2) e^{-s(l+1)^{2} / a^{2}} .
$$

The eigenstates of $\not D_{A d S_{2}}$ are given by the analytic continuation of the eigenfunctions given in (3.28)[103], making the replacement $\psi \rightarrow i \eta, l \rightarrow-i \lambda-1, \phi \rightarrow \theta$,

$$
\begin{aligned}
\chi_{m}^{ \pm}(\lambda)= & \frac{1}{\sqrt{4 \pi a^{2}}}\left|\frac{\Gamma(1+m+i \lambda)}{\Gamma(m+1) \Gamma\left(\frac{1}{2}+i \lambda\right)}\right| e^{i\left(m+\frac{1}{2}\right) \theta} \\
& \left(\begin{array}{c}
i \frac{\lambda}{m+1} \cosh ^{m} \frac{\eta}{2} \sinh ^{m+1} \frac{\eta}{2} F\left(m+1+i \lambda, m+1-i \lambda ; m+2 ;-\sinh ^{2} \frac{\eta}{2}\right) \\
\pm \cosh ^{m+1} \frac{\eta}{2} \sinh ^{m} \frac{\eta}{2} F\left(m+1+i \lambda, m+1-i \lambda ; m+1 ;-\sinh ^{2} \frac{\eta}{2}\right)
\end{array}\right), \\
\eta_{m}^{ \pm}(\lambda)= & \frac{1}{\sqrt{4 \pi a^{2}}}\left|\frac{\Gamma(1+m+i \lambda)}{\Gamma(m+1) \Gamma\left(\frac{1}{2}+i \lambda\right)}\right| e^{-i\left(m+\frac{1}{2}\right) \theta} \\
& \left(\begin{array}{c}
\cosh ^{m+1} \frac{\eta}{2} \sinh ^{m} \frac{\eta}{2} F\left(m+1+i \lambda, m+1-i \lambda ; m+1 ;-\sinh ^{2} \frac{\eta}{2}\right) \\
\pm i \frac{\lambda}{m+1} \cosh ^{m} \frac{\eta}{2} \sinh ^{m+1} \frac{\eta}{2} F\left(m+1+i \lambda, m+1-i \lambda ; m+2 ;-\sinh ^{2} \frac{\eta}{2}\right)
\end{array}\right), \\
& m \in \mathbb{Z}, \quad 0 \leq m<\infty, \quad 0<\lambda<\infty,
\end{aligned}
$$

satisfying

$$
\not D_{A d S_{2}} \chi_{m}^{ \pm}(\lambda)= \pm i a^{-1} \lambda \chi_{m}^{ \pm}(\lambda), \quad \not D_{A d S_{2}} \eta_{m}^{ \pm}(\lambda)= \pm i a^{-1} \lambda \eta_{m}^{ \pm}(\lambda)
$$

This gives

$$
\begin{aligned}
K_{A d S_{2}}^{f}(0 ; s)= & -\int_{0}^{\infty} d \lambda e^{-s \lambda^{2} / a^{2}} \\
& \sum_{m=0}^{\infty}\left[\left(\chi_{m}^{+}(\lambda)\right)^{\dagger} \chi_{m}^{+}(\lambda)+\left(\chi_{m}^{-}(\lambda)\right)^{\dagger} \chi_{m}^{-}(\lambda)+\left(\eta_{m}^{+}(\lambda)\right)^{\dagger} \eta_{m}^{+}(\lambda)+\left(\eta_{m}^{-}(\lambda)\right)^{\dagger} \eta_{m}^{-}(\lambda)\right] \\
= & -\frac{1}{\pi a^{2}} \int_{0}^{\infty} d \lambda e^{-\bar{s} \lambda^{2}} \lambda \operatorname{coth}(\pi \lambda) .
\end{aligned}
$$


In arriving at (3.36) we have evaluated $\chi_{m}^{ \pm}(\lambda), \eta_{m}^{ \pm}(\lambda)$ at $\eta=0$ since the final result is independent of the point in $A d S_{2}$ where we evaluate it.

The expansion of $K_{S^{2}}^{f}(s ; 0)$ and $K_{A d S_{2}}^{f}(s ; 0)$ for small $s$ can be found in the same way as for $K_{S^{2}}^{s}$ and $K_{A d S_{2}}^{s}$. We get

$$
\begin{aligned}
K_{A d S_{2}}^{f}(0 ; s) & =-\frac{1}{2 \pi a^{2} \bar{s}}\left[1+\sum_{n=0}^{\infty} \frac{(-1)^{n}}{n !}(2 n+1) ! \frac{\bar{s}^{n+1}}{\pi^{2 n+2}} \frac{1}{2^{2 n}} \zeta(2 n+2)\right] \\
& =-\frac{1}{2 \pi a^{2} \bar{s}}\left(1+\frac{1}{6} \bar{s}-\frac{1}{60} \bar{s}^{2}+\mathcal{O}\left(\bar{s}^{3}\right)\right),
\end{aligned}
$$

and

$$
\begin{aligned}
K_{S^{2}}^{s}(0 ; s) & =-\frac{1}{2 \pi a^{2} \bar{s}}\left[1-\sum_{n=0}^{\infty} \frac{1}{n !}(2 n+1) ! \frac{\bar{s}^{n+1}}{\pi^{2 n+2}} \frac{1}{2^{2 n}} \zeta(2 n+2)\right] \\
& =-\frac{1}{2 \pi a^{2} \bar{s}}\left(1-\frac{1}{6} \bar{s}-\frac{1}{60} \bar{s}^{2}+\mathcal{O}\left(\bar{s}^{3}\right)\right) .
\end{aligned}
$$

Now suppose that $\psi_{1}$ denotes an eigenstate of $\not D_{S^{2}}$ with eigenvalue $i \tilde{\kappa}_{1}$ and $\psi_{2}$ denotes an eigenstate of $\not D_{A d S_{2}}$ with eigenvalue $i \tilde{\kappa}_{2}$ :

$$
\not D_{S^{2}} \psi_{1}=i \tilde{\kappa}_{1} \psi_{1}, \quad \not D_{A d S_{2}} \psi_{2}=i \tilde{\kappa}_{2} \psi_{2}
$$

Since $\sigma_{3}$ anti-commutes with $\not D_{S^{2}}$ and commutes with $\not D_{A d S_{2}}$, we have, using (3.25),

$$
\begin{aligned}
\not D_{A d S_{2} \times S^{2}} \psi_{1} \otimes \psi_{2} & =i \tilde{\kappa}_{1} \psi_{1} \otimes \psi_{2}+i \tilde{\kappa}_{2} \sigma_{3} \psi_{1} \otimes \psi_{2}, \\
\not D_{A d S_{2} \times S^{2}} \sigma_{3} \psi_{1} \otimes \psi_{2} & =i \tilde{\kappa}_{2} \psi_{1} \otimes \psi_{2}-i \tilde{\kappa}_{1} \sigma_{3} \psi_{1} \otimes \psi_{2} .
\end{aligned}
$$

Diagonalizing the $2 \times 2$ matrix we see that $\not D_{A d S_{2} \times S^{2}}$ has eigenvalues $\pm i \sqrt{\tilde{\kappa}_{1}^{2}+\tilde{\kappa}_{2}^{2}}$. Thus the square of the eigenvalue of $\not D_{A d S_{2} \times S^{2}}$ is given by the sum of squares of the eigenvalues of $\not D_{A d S_{2}}$ and $\not D_{S^{2}}$. This in turn gives

$$
K_{A d S_{2} \times S^{2}}^{f}=-K_{A d S_{2}}^{f} K_{S^{2}}^{f},
$$

where the minus sign again accounts for the fact that the fermionic integration produces a factor of the determinant instead of the inverse of the determinant. Using (3.37) and (3.38) we get

$$
K_{A d S_{2} \times S^{2}}^{f}=-\frac{1}{4 \pi^{2} a^{4} \bar{s}^{2}}\left(1-\frac{11}{180} \bar{s}^{2}+O\left(\bar{s}^{3}\right)\right) .
$$

The $s$ independent term in this expression is in agreement with the results of [100].

\section{Effect of graviphoton background in $\mathcal{N}=4$ supersymmetric string theory}

Quarter BPS black holes in $\mathcal{N}=4$ supersymmetric string theories, obtained by compactifying heterotic string theory on $T^{6}$ or equivalently type II string theory on $K 3 \times T^{2}$, have near horizon $A d S_{2} \times S^{2}$ geometry. The background is also accompanied by flux of electromagnetic fields along $A d S_{2}$ and $S^{2}$. The presence of this flux modifies the kinetic terms of various fields around this background, and hence also the associated heat kernels. In this section we shall compute the modification of the kinetic term of various fields due to these fluxes. 


\subsection{Four dimensional $\mathcal{N}=4$ supergravity from ten dimensional $\mathcal{N}=1$ super- gravity}

We shall begin by reviewing the dimensional reduction of the ten dimensional supergravity action on $T^{6}$ leading to the four dimensional $\mathcal{N}=4$ supergravity action. ${ }^{13}$ The action of $\mathcal{N}=1$ supergravity in ten dimensions coupled to 16 Maxwell fields is given by [110]:

$$
\begin{aligned}
& \frac{1}{(2 \pi)^{6}\left(\alpha^{\prime}\right)^{4}} \int d^{10} z \sqrt{\operatorname{det} G^{(10)}} e^{-2 \Phi^{(10)}}\left[R^{(10)}+\frac{1}{4} G^{(10) M N} \partial_{M} \Phi^{(10)} \partial_{N} \Phi^{(10)}\right. \\
& -\frac{1}{12} H_{M N P}^{(10)} H^{(10) M N P}-\frac{1}{4} F_{M N}^{(10) I} F^{(10) I M N} \\
& -\left\{\frac{1}{2} \bar{\psi}_{M}^{(10)} \Gamma^{M N P} D_{N} \psi_{P}^{(10)}+\frac{1}{2} \bar{\Lambda} \Gamma^{M} D_{M} \Lambda+\frac{1}{2} \bar{\Sigma}^{I} \Gamma^{M} D_{M} \Sigma^{I}\right. \\
& -\frac{1}{48} \bar{\Sigma}^{I} \Gamma^{M N P} \Sigma^{I} H_{M N P}^{(10)}+\frac{1}{2 \sqrt{2}} \bar{\Sigma}^{I} \Gamma^{M} \Gamma^{N P}\left(\psi_{M}^{(10)}+\frac{1}{6 \sqrt{2}} \Gamma_{M} \Lambda\right) F_{N P}^{(10) I} \\
& \left.\left.-\frac{1}{48}\left(\bar{\psi}_{M}^{(10)} \Gamma^{M N P Q R} \psi_{R}^{(10)}+6 \bar{\psi}^{(10) N} \Gamma^{P} \psi^{(10) Q}-\sqrt{2} \bar{\psi}_{M}^{(10)} \Gamma^{N P Q} \Gamma^{M} \Lambda\right) H_{N P Q}^{(10)}\right\}\right] \\
& +\cdots .
\end{aligned}
$$

Here $G_{M N}^{(10)}, B_{M N}^{(10)}, A_{M}^{(10) I}$, and $\Phi^{(10)}$ are ten dimensional metric, anti-symmetric tensor field, $\mathrm{U}(1)$ gauge fields and the scalar dilaton field respectively $(0 \leq M, N \leq 9,1 \leq I \leq 16), \psi_{M}^{(10)}$ denotes a left-handed Majorana-Weyl gravitino field, $\Lambda$ is a right-handed Majorana-Weyl spinor field and $\Sigma^{I}$ are left-handed Majorana-Weyl spinor fields in the gauge multiplet. ... denotes terms containing fermion bilinears multiplied by derivatives of the dilaton or terms quartic in the fermions, and

$$
\begin{aligned}
F_{M N}^{(10) I} & =\partial_{M} A_{N}^{(10) I}-\partial_{N} A_{M}^{(10) I} \\
H_{M N P}^{(10)} & =\left(\partial_{M} B_{N P}^{(10)}-\frac{1}{2} A_{M}^{(10) I} F_{N P}^{(10) I}\right)+\text { cyclic permutations in } M, N, P, \\
D_{M} \psi_{P}^{(10)} & =\partial_{M} \psi_{P}^{(10)}-\left\{\begin{array}{c}
N \\
M P
\end{array}\right\} \psi_{N}^{(10)}+\frac{1}{4} \omega_{M}^{A B} \Gamma^{A B} \psi_{P}^{(10)}, \\
D_{M}\left(\begin{array}{c}
\Sigma^{I} \\
\Lambda
\end{array}\right) & =\left(\partial_{M}+\frac{1}{4} \omega_{M}^{A B} \Gamma^{A B}\right)\left(\begin{array}{c}
\Sigma^{I} \\
\Lambda
\end{array}\right), \\
\omega_{M}^{A B} & =-G^{(10) N P} e_{N}^{B} \partial_{M} e_{P}^{A}+e_{N}^{A} e_{P}^{B} G^{(10) P Q}\left\{\begin{array}{c}
N \\
Q M
\end{array}\right\}, \\
\left\{\begin{array}{c}
M \\
N P
\end{array}\right\} & =\frac{1}{2} G^{(10) M R}\left(\partial_{N} G_{P R}^{(10)}+\partial_{P} G_{N R}^{(10)}-\partial_{R} G_{N P}^{(10)}\right),
\end{aligned}
$$

the $e_{M}^{A}$ being the vielbeins. $\Gamma^{A}$ 's are the $32 \times 32 \mathrm{SO}(10)$ gamma matrices, $\Gamma^{A B} \equiv\left(\Gamma^{A} \Gamma^{B}-\right.$ $\left.\Gamma^{B} \Gamma^{A}\right) / 2$, and

$$
\bar{\psi}_{M}^{(10)} \equiv \psi_{M}^{(10) T} C, \quad \bar{\Lambda} \equiv \Lambda^{T} C, \quad \bar{\Sigma}^{I} \equiv \Sigma^{I T} C,
$$

\footnotetext{
${ }^{13}$ We could have directly began with the $\mathcal{N}=4$ supergravity action in four dimensions given in (4.20). However for dealing with the fermions we have found it more convenient to use the ten dimensional description.
} 
where $T$ denotes transpose and $C$ is the $\mathrm{SO}(10)$ charge conjugation matrix satisfying

$$
\left(C \Gamma^{A}\right)^{T}=C \Gamma^{A} .
$$

We can use the vielbeins to convert the tangent space indices to coordinate indices and vice versa. We shall use the same symbol $\Gamma$ for labelling the gamma matrices carrying coordinate indices. Our choice for the ten dimensional gamma matrices and the charge conjugation matrix will be as follows:

$$
\begin{aligned}
& \Gamma^{0}=-\sigma_{3} \otimes \tau_{2} \otimes I_{8}, \quad \Gamma^{1}=\sigma_{3} \otimes \tau_{1} \otimes I_{8}, \quad \Gamma^{2}=-\sigma_{2} \otimes I_{2} \otimes I_{8}, \quad \Gamma^{3}=\sigma_{1} \otimes I_{2} \otimes I_{8}, \\
& \Gamma^{p}=\sigma_{3} \otimes \tau_{3} \otimes \widehat{\Gamma}^{p}, \quad C=\sigma_{2} \otimes \tau_{1} \otimes \widehat{C}, \quad 4 \leq p \leq 9,
\end{aligned}
$$

where $\widehat{\Gamma}^{p}$ are $8 \times 8 \mathrm{SO}(6)$ gamma matrices and $\widehat{C}$ is the $\mathrm{SO}(6)$ charge conjugation matrix satisfying

$$
\left\{\widehat{\Gamma}^{p}, \widehat{\Gamma}^{q}\right\}=2 \delta_{p q}, \quad\left(\widehat{C} \widehat{\Gamma}^{p}\right)^{T}=-\widehat{C} \widehat{\Gamma}^{p}, \quad \widehat{C}^{T}=\widehat{C} .
$$

The spinors are taken to be 32 component, and the Weyl condition is imposed by setting to zero half of these components. Finally note that in ten euclidean dimensions we cannot impose Majorana-Weyl condition and hence must formally allow the spinors to be complex. However we shall continue to use (4.4) as the definition of the barred fields. As a result the action will not be real.

The supersymmetry transformation laws of various fields can be found in the standard literature (see e.g. [110]). We shall only need to know the supersymmetry transformation laws of $\psi_{M}^{(10)}$ and $\Lambda$. In the background where all scalar fields are constants this has the form:

$$
\delta \psi_{M}^{(10)}=D_{M} \eta+\frac{1}{96}\left(\Gamma_{M}^{N P Q}-9 \delta_{M}^{N} \Gamma^{P Q}\right) H_{N P Q} \eta, \quad \delta \Lambda=\frac{1}{24 \sqrt{2}} \Gamma^{M N P} H_{M N P} \eta,
$$

where $\eta$ is the supersymmetry transformation parameter.

For dimensional reduction, it is convenient to introduce the 'four dimensional fields' $\widehat{G}_{\bar{a} \bar{b}}, \widehat{B}_{\bar{a} \bar{b}}, \widehat{A}_{\bar{a}}^{I}, \Phi, A_{\mu}^{i}, G_{\mu \nu}$ and $B_{\mu \nu}$ for $4 \leq \bar{a}, \bar{b} \leq 9,0 \leq \mu, \nu \leq 3,1 \leq I \leq 16$ and $1 \leq i \leq 28$ through the relations $[111,112]$

$$
\begin{array}{rlrl}
\widehat{G}_{\bar{a} \bar{b}} & =G_{\bar{a} \bar{b}}^{(10)}, & \widehat{B}_{\bar{a} \bar{b}}=B_{\bar{a} \bar{b}}^{(10)}, & \widehat{A}_{\bar{a}}^{I}=A_{\bar{a}}^{(10) I}, \\
A_{\mu}^{\bar{a}-3} & =\frac{1}{2} \widehat{G}^{\bar{a} \bar{b}} G_{\bar{b} \mu}^{(10)}, & A_{\mu}^{I+12}=-\left(\frac{1}{2} A_{\mu}^{(10) I}-\widehat{A}_{\bar{b}}^{I} A_{\mu}^{\bar{b}-3}\right), \\
A_{\mu}^{\bar{a}+3} & =\frac{1}{2} B_{\bar{a} \mu}^{(10)}-\widehat{B}_{\bar{a} \bar{b}} A_{\mu}^{\bar{b}-3}+\frac{1}{2} \widehat{A}_{\bar{a}}^{I} A_{\mu}^{I+12}, \\
G_{\mu \nu} & =G_{\mu \nu}^{(10)}-G_{\bar{a} \mu}^{(10)} G_{\bar{b} \nu}^{(10)} \widehat{G}^{\bar{a} \bar{b}}, \\
B_{\mu \nu} & =B_{\mu \nu}^{(10)}-4 \widehat{B}_{\bar{a} \bar{b}} A_{\mu}^{\bar{a}-3} A_{\nu}^{\bar{b}-3}-2\left(A_{\mu}^{\bar{a}-3} A_{\nu}^{\bar{a}+3}-A_{\nu}^{\bar{a}-3} A_{\mu}^{\bar{a}+3}\right), \\
\Phi & =\Phi^{(10)}-\frac{1}{4} \ln \operatorname{det} \widehat{G}, \\
\widehat{\psi}_{\bar{a}} & =\psi_{\bar{a}}^{(10)}, & \widetilde{\psi}_{\mu}=\psi_{\mu}^{(10)}-2 \widehat{\psi}_{\bar{a}} A_{\mu}^{\bar{a}-3} .
\end{array}
$$


Here $\widehat{G}^{\bar{a} \bar{b}}$ denotes the inverse of the matrix $\widehat{G}_{\bar{a} \bar{b}}$. We have not displayed the spinor indices explicitly; it is enough to note that under this dimensional reduction the spinor representation of the ten dimensional rotation group splits into a product of a spinor representation of the four dimensional rotation group and a spinor representation of the $\mathrm{SO}(6) \mathrm{R}$-symmetry group. We now combine the scalar fields $\widehat{G}_{\bar{a} \bar{b}}, \widehat{B}_{\bar{a} \bar{b}}$, and $\widehat{A}_{\bar{a}}^{I}$ into an $O(6,22)$ matrix valued scalar field $M$. For this we regard $\widehat{G}_{\bar{a} \bar{b}}, \widehat{B}_{\bar{a} \bar{b}}$ and $\widehat{A}_{\bar{a}}^{I}$ as $6 \times 6,6 \times 6$, and $6 \times 16$ matrices respectively, $\widehat{C}_{\bar{a} \bar{b}}=\frac{1}{2} \widehat{A}_{\bar{a}}^{I} \widehat{A}_{\bar{b}}^{I}$ as a $6 \times 6$ matrix, and define $M$ to be the $28 \times 28$ dimensional matrix

$$
M=\left(\begin{array}{ccc}
\widehat{G}^{-1} & \widehat{G}^{-1}(\widehat{B}+\widehat{C}) & \widehat{G}^{-1} \widehat{A} \\
(-\widehat{B}+\widehat{C}) \widehat{G}^{-1} & (\widehat{G}-\widehat{B}+\widehat{C}) \widehat{G}^{-1}(\widehat{G}+\widehat{B}+\widehat{C}) & (\widehat{G}-\widehat{B}+\widehat{C}) \widehat{G}^{-1} \widehat{A} \\
\widehat{A}^{T} \widehat{G}^{-1} & \widehat{A} \widehat{G}^{-1}(\widehat{G}+\widehat{B}+\widehat{C}) & I_{16}+\widehat{A}^{T} \widehat{G}^{-1} \widehat{A}
\end{array}\right)
$$

$M$ satisfies

$$
M L M^{T}=L, \quad M^{T}=M, \quad L=\left(\begin{array}{ccc}
0 & I_{6} & 0 \\
I_{6} & 0 & 0 \\
0 & 0 & -I_{16}
\end{array}\right)
$$

where $I_{n}$ denotes the $n \times n$ identity matrix.

The effective action that governs the dynamics of the massless fields in the four dimensional theory is obtained by substituting the expressions for the ten dimensional fields in terms of the four dimensional fields in eq. (4.1), and taking all field configurations to be independent of the internal coordinates. The result is

$$
\begin{gathered}
S=\frac{1}{2 \pi \alpha^{\prime}} \int d^{4} x \sqrt{\operatorname{det} G} e^{-2 \Phi}\left[R_{G}+4 G^{\mu \nu} \partial_{\mu} \Phi \partial_{\nu} \Phi-\frac{1}{12} G^{\mu \mu^{\prime}} G^{\nu \nu^{\prime}} G^{\rho \rho^{\prime}} H_{\mu \nu \rho} H_{\mu^{\prime} \nu^{\prime} \rho^{\prime}}\right. \\
\left.-G^{\mu \mu^{\prime}} G^{\nu \nu^{\prime}} F_{\mu \nu}^{i}(L M L)_{i j} F_{\mu^{\prime} \nu^{\prime}}^{j}+\frac{1}{8} G^{\mu \nu} \operatorname{Tr}\left(\partial_{\mu} M L \partial_{\nu} M L\right)\right]+S_{f}
\end{gathered}
$$

where $S_{f}$ denotes the fermionic terms, $R_{G}$ is the scalar curvature associated with the four dimensional metric $G_{\mu \nu}$, and

$$
\begin{aligned}
F_{\mu \nu}^{i} & =\partial_{\mu} A_{\nu}^{i}-\partial_{\nu} A_{\mu}^{i} \\
H_{\mu \nu \rho} & =\left(\partial_{\mu} B_{\nu \rho}+2 A_{\mu}^{i} L_{i j} F_{\nu \rho}^{j}\right)+\text { cyclic permutations of } \mu, \nu, \rho .
\end{aligned}
$$

In deriving this result we have taken $\int d^{6} y=\left(2 \pi \sqrt{\alpha^{\prime}}\right)^{6}$, where $y^{m}(1 \leq m \leq 6)$ denote the coordinates labeling the six dimensional torus. Note that we have not written down the fermionic terms explicitly. Instead of writing the four dimensional action involving the fermions we shall find it more convenient to evaluate the quadratic term in the fermions in the black hole background by directly using the ten dimensional action (4.1).

For our analysis it will be convenient to use a new set of field variables which are related to the ones described above by a rotation in the internal space. We define

$$
U=\left(\begin{array}{rrr}
I_{6} / \sqrt{2} & I_{6} / \sqrt{2} \\
-I_{6} / \sqrt{2} & I_{6} / \sqrt{2} & \\
& & I_{16}
\end{array}\right)
$$


and

$$
\bar{A}_{\mu}^{i}=U_{i j} A_{\mu}^{j}, \quad \bar{M}=U M U^{-1}, \quad \bar{L}=U L U^{-1}=\left(\begin{array}{cc}
I_{6} & \\
& -I_{22}
\end{array}\right), \quad \bar{F}_{\mu \nu}^{i}=U_{i j} F_{\mu \nu}^{j},
$$

so that

$$
\bar{M} \bar{L} \bar{M}^{T}=\bar{L}, \quad \bar{M}^{T}=\bar{M} .
$$

In these new variables the action takes the form:

$$
\begin{aligned}
& S=\frac{1}{2 \pi \alpha^{\prime}} \int d^{4} x \sqrt{\operatorname{det} G} e^{-2 \Phi}\left[R_{G}+4 G^{\mu \nu} \partial_{\mu} \Phi \partial_{\nu} \Phi-\frac{1}{12} G^{\mu \mu^{\prime}} G^{\nu \nu^{\prime}} G^{\rho \rho^{\prime}} H_{\mu \nu \rho} H_{\mu^{\prime} \nu^{\prime} \rho^{\prime}}\right. \\
&\left.-G^{\mu \mu^{\prime}} G^{\nu \nu^{\prime}} \bar{F}_{\mu \nu}^{i}(\bar{L} \bar{M} \bar{L})_{i j} \bar{F}_{\mu^{\prime} \nu^{\prime}}^{j}+\frac{1}{8} G^{\mu \nu} \operatorname{Tr}\left(\partial_{\mu} \bar{M} \bar{L} \partial_{\nu} \bar{M} \bar{L}\right)\right]+S_{f} \\
& H_{\mu \nu \rho}=\left(\partial_{\mu} B_{\nu \rho}+2 \bar{A}_{\mu}^{i} \bar{L}_{i j} \bar{F}_{\nu \rho}^{j}\right)+\text { cyclic permutations of } \mu, \nu, \rho
\end{aligned}
$$

Finally we can arrive at a simpler version of the action by dualizing the 3 -form field via the relation

$$
H^{\mu \nu \rho}=-i(\sqrt{\operatorname{det} G})^{-1} e^{2 \Phi} \epsilon^{\mu \nu \rho \sigma} \partial_{\sigma} \Psi
$$

where $\Psi$ is a scalar field. The new action is then given by

$$
\begin{aligned}
S= & \frac{1}{2 \pi \alpha^{\prime}} \int d^{4} x \sqrt{\operatorname{det} G} e^{-2 \Phi}\left[R_{G}+4 G^{\mu \nu} \partial_{\mu} \Phi \partial_{\nu} \Phi-\frac{1}{2} e^{4 \Phi} G^{\mu \nu} \partial_{\nu} \Psi \partial_{\mu} \Psi\right. \\
& -G^{\mu \mu^{\prime}} G^{\nu \nu^{\prime}} \bar{F}_{\mu \nu}^{i}(\bar{L} \bar{M} \bar{L})_{i j} \bar{F}_{\mu^{\prime} \nu^{\prime}}^{j}+\Psi e^{2 \Phi} G^{\mu \mu^{\prime}} G^{\nu \nu^{\prime}} \bar{F}_{\mu \nu}^{i} \bar{L}_{i j} \widetilde{\bar{F}}_{\mu^{\prime} \nu^{\prime}}^{j} \\
& \left.+\frac{1}{8} G^{\mu \nu} \operatorname{Tr}\left(\partial_{\mu} \bar{M} \bar{L} \partial_{\nu} \bar{M} \bar{L}\right)\right]+S_{f}, \\
\widetilde{\bar{F}}^{i \mu \nu} \equiv & \frac{i}{2}(\sqrt{\operatorname{det} G})^{-1} \epsilon^{\mu \nu \rho \sigma} \bar{F}_{\rho \sigma}^{i} .
\end{aligned}
$$

\subsection{The quadratic action for the fluctuations around the attractor geometry}

We shall consider black holes carrying (electric,magnetic) charge vectors ${ }^{14}$

$$
\bar{Q}=\left(\begin{array}{c}
Q_{0} \\
0 \\
\cdot \\
\cdot \\
\cdot \\
0
\end{array}\right), \quad \bar{P}=\left(\begin{array}{c}
0 \\
P_{0} \\
\cdot \\
\cdot \\
\cdot \\
0
\end{array}\right)
$$

\footnotetext{
${ }^{14}$ While this is a very specific choice of the charges, and pair of charges $(\bar{Q}, \bar{P})$, satisfying $\bar{Q}^{2} \equiv \bar{Q}^{T} \bar{L} \bar{Q}>0$, $\bar{P}^{2}>0$ and $\bar{Q}^{2} \bar{P}^{2}>(\bar{Q} \cdot \bar{P})^{2}$, can be brought to this form with the help of a continuous $\operatorname{SL}(2, \mathbb{R}) \times O(6,22)$ transformation which is a symmetry of the supergravity equations of motion. Thus the final result of our analysis holds for any $(\bar{Q}, \bar{P})$ satisfying $\bar{Q}^{2}>0, \bar{P}^{2}>0, \bar{Q}^{2} \bar{P}^{2}>(\bar{Q} \cdot \bar{P})^{2},-$ conditions under which a supersymmetric black hole solution exists.
} 
Then in an appropriate normalization convention the near horizon geometry is given by [29]:

$$
\begin{aligned}
& d s^{2}=a^{2}\left(d \eta^{2}+\sinh ^{2} \eta d \theta^{2}\right)+a^{2}\left(d \psi^{2}+\sin ^{2} \psi d \phi^{2}\right), \quad a^{2}=\frac{\alpha^{\prime}}{8} P_{0}^{2}, \quad e^{-2 \Phi}=\frac{Q_{0}}{P_{0}}, \quad \bar{M}=I_{28} \\
& \bar{F}_{\eta \theta}^{i}=-i \frac{\sqrt{\alpha^{\prime}}}{4} \sinh \eta \frac{P_{0}}{Q_{0}} \bar{Q}_{i}, \quad \bar{F}_{\psi \phi}^{i}=\frac{\sqrt{\alpha^{\prime}}}{4} \bar{P}_{i} \sin \psi, \quad H_{\mu \nu \rho}=0 .
\end{aligned}
$$

We shall make the choice of vierbeins given in (3.23) and denote the indices labelling the coordinates of $S^{2}$ by $\alpha, \beta, \cdots$, the indices labelling the coordinates of $A d S_{2}$ by $m, n, \cdots$ and the indices labelling all the four cordinates by $\mu, \nu, \cdots$.

We shall study quadratic fluctuations of various fields around the background (4.23). Let us denote the background values of various fields given in (4.23) by the superscript (0). We parametrize the bosonic fluctuations as follows:

$$
\Phi=\Phi^{(0)}+\frac{1}{2} \chi_{2}, \quad G_{\mu \nu}=e^{\chi_{2}}\left\{G_{\mu \nu}^{(0)}+h_{\mu \nu}\right\}, \quad \Psi=\frac{Q_{0}}{P_{0}} \chi_{1}, \quad \bar{A}_{\mu}^{i}=\bar{A}_{\mu}^{i(0)}+\frac{1}{2} \mathcal{A}_{\mu}^{(i)} .
$$

Special care is taken to parametrize $\bar{M}$ since it is a constrained field. A parametrization satisfying (4.16) to quadratic order in the fluctuations is as follows:

$\bar{M}_{a r}=\bar{M}_{r a}=\sqrt{2} \phi_{a r}, \quad \bar{M}_{a b}=\delta_{a b}+\phi_{a r} \phi_{b r}, \quad \bar{M}_{r s}=\delta_{r s}+\phi_{a r} \phi_{a s}, \quad 1 \leq a, b \leq 6, \quad 7 \leq r, s \leq 28$.

We also need to add to the action the gauge fixing term:

$$
\begin{aligned}
& \frac{1}{2 \pi \alpha^{\prime}} \frac{Q_{0}}{P_{0}} \int d^{4} x \sqrt{\operatorname{det} G^{(0)}} \mathcal{L}_{g f}, \\
\mathcal{L}_{g f}= & -\frac{1}{2} g^{\rho \sigma}\left(D^{\mu} h_{\mu \rho}-\frac{1}{2} D_{\rho} h^{\mu}{ }_{\mu}\right)\left(D^{\nu} h_{\nu \sigma}-\frac{1}{2} D_{\sigma} h^{\nu}{ }_{\nu}\right)-\frac{1}{2} D^{\mu} \mathcal{A}_{\mu}^{(i)} D^{\nu} \mathcal{A}_{\nu}^{(i)},
\end{aligned}
$$

where all covariant derivatives and raising of lowering of indices are computed with the background $A d S_{2} \times S^{2}$ metric. Substituting (4.24), (4.25) into (4.20), adding to it (4.26) and expanding it to quadratic order in the fluctuations we get

$$
S=S^{(0)}+\frac{1}{2 \pi \alpha^{\prime}} \frac{Q_{0}}{P_{0}} \int d^{4} x \sqrt{\operatorname{det} G^{(0)}}\left[\mathcal{L}_{\text {standard }}+\mathcal{L}_{\text {flux }}\right]+S_{f}
$$

Here $\mathcal{L}_{\text {standard }}$ is the standard gauge fixed action for various free quantum fields in the $A d S_{2} \times S^{2}$ background metric:

$$
\begin{aligned}
\mathcal{L}_{\text {standard }}= & -\frac{1}{4} h_{\mu \nu}(\widetilde{\Delta} h)^{\mu \nu}+\frac{1}{2} \chi_{1} \square \chi_{1}+\frac{1}{2} \chi_{2} \square \chi_{2}+\frac{1}{2} \sum_{a=1}^{6} \mathcal{A}_{\mu}^{(a)}\left(G^{(0) \mu \nu} \square-R^{\mu \nu}\right) \mathcal{A}_{\nu}^{(a)} \\
& +\frac{1}{2} \sum_{r=7}^{28} \mathcal{A}_{\mu}^{(r)}\left(G^{(0) \mu \nu} \square-R^{\mu \nu}\right) \mathcal{A}_{\nu}^{(r)}+\frac{1}{2} \sum_{a=1}^{6} \sum_{r=7}^{28} \phi_{a r} \square \phi_{a r},
\end{aligned}
$$

where,

$$
\begin{aligned}
(\widetilde{\Delta} h)_{\mu \nu}= & -\square h_{\mu \nu}-R_{\mu \tau} h_{\nu}^{\tau}-R_{\nu \tau} h_{\mu}^{\tau}-2 R_{\mu \rho \nu \tau} h^{\rho \tau}+\frac{1}{2} G_{\mu \nu}^{(0)} G^{(0) \rho \sigma} \square h_{\rho \sigma} \\
& +R h_{\mu \nu}+\left(g_{\mu \nu} R^{\rho \sigma}+R_{\mu \nu} g^{\rho \sigma}\right) h_{\rho \sigma}-\frac{1}{2} R g_{\mu \nu} g^{\rho \sigma} h_{\rho \sigma}
\end{aligned}
$$

Here all indices are lowered and raised by the background metric $G_{\mu \nu}^{(0)}$ and its inverse, and $R_{\mu \nu \rho \sigma}$, $R_{\mu \nu}$ and $R$ are calculated with the metric $G_{\mu \nu}^{(0)} . \mathcal{L}_{\text {flux }}$ denotes the extra terms due to background 
flux:

$$
\begin{aligned}
\mathcal{L}_{\text {flux }}= & 2 a^{-1} \phi_{2 r} \varepsilon^{\gamma \beta} \partial_{\gamma} \mathcal{A}_{\beta}^{(r)}-a^{-2} \phi_{2 r} \phi_{2 r}-2 i a^{-1} \phi_{1 r} \varepsilon^{m n} \partial_{m} \mathcal{A}_{n}^{(r)}+a^{-2} \phi_{1 r} \phi_{1 r} \\
& +\frac{1}{2} a^{-2}\left(h^{m n} h_{m n}-h^{\alpha \beta} h_{\alpha \beta}+2 \chi_{2}\left(h_{m}^{m}-h_{\alpha}^{\alpha}\right)\right)+\frac{\sqrt{2}}{a}\left[i \varepsilon^{m n} f_{\alpha m}^{(1)} h_{n}^{\alpha}+\varepsilon^{\alpha \beta} f_{\alpha m}^{(2)} h_{\beta}{ }^{m}\right] \\
& +\frac{1}{2 \sqrt{2} a}\left[i \varepsilon^{m n} f_{m n}^{(1)}\left(-2 \chi_{2}+h_{\gamma}^{\gamma}-h^{p}{ }_{p}\right)-\varepsilon^{\alpha \beta} f_{\alpha \beta}^{(2)}\left(-2 \chi_{2}+h^{p}{ }_{p}-h_{\gamma}^{\gamma}\right)\right] \\
& +\frac{1}{a \sqrt{2}} \chi_{1}\left(i \varepsilon^{m n} f_{m n}^{(2)}+\varepsilon^{\alpha \beta} f_{\alpha \beta}^{(1)}\right) \\
f_{\mu \nu}^{(i)} \equiv & \partial_{\mu} \mathcal{A}_{\nu}^{(i)}-\partial_{\nu} \mathcal{A}_{\mu}^{(i)} .
\end{aligned}
$$

Here $\varepsilon^{\alpha \beta}$ and $\varepsilon^{m n}$ are the invariant antisymmetric tensors on $S^{2}$ and $A d S_{2}$ respectively, computed with the background metric $G_{\mu \nu}^{(0)}$ :

$$
\varepsilon_{\psi \phi}=a^{2} \sin \psi, \quad \varepsilon_{\eta \theta}=a^{2} \sinh \eta .
$$

$\mathcal{L}_{\text {standard }}$ is what was used in section 3 for computing the heat kernel of various fields; so our main goal will be to compute the effect of $\mathcal{L}_{\text {flux }}$ on the heat kernels.

Note that both $\mathcal{L}_{\text {standard }}$ and $\mathcal{L}_{\text {flux }}$ have an $\mathrm{SO}(22)$ symmetry acting on the index $r$. This is a remnant of the $\mathrm{SO}(6,22)$ continuous duality symmetry of the original supergravity action (4.17) of which an $\mathrm{SO}(4,22)$ subgroup survives in the background (4.23). The gauge fixing term (4.26) also respects this symmetry. The various fluctuations transform either as a singlet or a vector of $\mathrm{SO}(22)$. We shall call the singlets of $\mathrm{SO}(22)$ fields in the gravity multiplet and the vectors of $\mathrm{SO}(22)$ fields in the matter multiplet. Clearly we can analyze separately the heat kernels from fields in the matter multiplet and the gravity multiplet since they will not mix at the quadratic level. In this paper we shall focus on the contribution from the matter multiplets only.

To this action we must also add the action for the ghosts associated with diffeomorphism and $\mathrm{U}(1)$ gauge invariances. Let us denote by $b_{\mu}$ and $c_{\mu}$ the ghosts associated with diffeomorphism invariance, by $b^{(i)}$ and $c^{(i)}$ the ghosts associated with the $\mathrm{U}(1)$ gauge invariances, and by

$$
\mathcal{F}_{\mu} \equiv D^{\rho} h_{\mu \rho}-\frac{1}{2} D_{\mu} h^{\rho}{ }_{\rho}, \quad \mathcal{F}^{(i)} \equiv D^{\rho} \mathcal{A}_{\rho}^{(i)}
$$

the gauge fixing terms for the diffeomorphism and $\mathrm{U}(1)$ gauge invariances. Then by standard rules the ghost lagrangian density will be given by

$$
b^{\mu} \delta \mathcal{F}_{\mu}++b^{(i)} \delta \mathcal{F}^{(i)},
$$

where $\delta$ denotes the variation under a diffeomorphism transformation with parameter $c_{\nu}$ and gauge transformations with parameters $c^{(i)}$. In the attractor geometry given in (4.23) we have

$$
\delta h_{\mu \nu}=D_{\mu} c_{\nu}+D_{\nu} c_{\mu}+\cdots, \quad \delta \mathcal{A}_{\mu}^{(i)}=D_{\mu} c^{(i)}-2 \bar{F}_{\mu \nu}^{i} c^{\nu}+2 D_{\mu}\left(\bar{A}_{\rho}^{(i)} c^{\rho}\right)+\cdots,
$$

where $D_{\mu}$ denotes covariant derivative computed using the background metric of the attractor geometry, $\bar{A}_{\mu}^{(i)}$ is the background gauge field (note the normalization factor of 2 between the background and fluctuations given in (4.24)) and $\cdots$ denotes terms higher order in the fluctuations. Using (4.32)-(4.34), and the fact that the background geometry satisfies $D^{\mu} \bar{F}_{\mu \nu}^{i}=0$, we see that the quadratic part of the ghost action is given by

$$
b^{\mu}\left(g_{\mu \nu} \square+R_{\mu \nu}\right) c^{\nu}+b^{(i)} \square c^{(i)}-2 b^{(i)} \bar{F}_{\mu \nu}^{i} D^{\mu} c^{\nu}+2 b^{(i)} \square\left(\bar{A}_{\rho}^{i} c^{\rho}\right)
$$

The last term can be removed by a field redefinition $c^{(i)} \rightarrow c^{(i)}-2 \bar{A}_{\rho}^{i} c^{\rho}$. This yields a simpler version of the ghost lagrangian density:

$$
\mathcal{L}_{\text {ghost }} \propto\left[b^{\mu}\left(g_{\mu \nu} \square+R_{\mu \nu}\right) c^{\nu}+b^{(i)} \square c^{(i)}-2 b^{(i)} \bar{F}_{\mu \nu}^{i} D^{\mu} c^{\nu}\right] .
$$


Note that the ghosts $\left(b^{(i)}, c^{(i)}\right)$ for $i=7, \cdots 28$ are $\mathrm{SO}(22)$ vectors and the rest of the ghosts are $\mathrm{SO}(22)$ singlets. As in the case of matter fields, we shall analyze the contribution from $\mathrm{SO}(22)$ vector ghosts only.

Finally let us turn to the fermionic terms in the action. In order to simplify the structure of the action we need to carry out a set of field redefinitions. We define

$$
\begin{aligned}
\psi_{\mu} & =\widetilde{\psi}_{\mu}+\frac{1}{2} \Gamma_{\mu} \Gamma^{\bar{a}} \widehat{\psi}_{\bar{a}}, \\
\lambda & =\frac{1}{2}\left(\Lambda+\sqrt{2} \Gamma^{\bar{a}} \widehat{\psi}_{\bar{a}}\right), \\
\varphi_{\bar{a}+3} & =\widehat{\psi}_{\bar{a}}-\frac{1}{2 \sqrt{2}} \Gamma_{\bar{a}} \Lambda, \\
\varphi_{I+12} & =\Sigma^{I}, \quad 0 \leq \mu, \nu \leq 3, \quad 4 \leq \bar{a}, \bar{b} \leq 9, \quad 1 \leq I \leq 16,
\end{aligned}
$$

where $\widehat{\psi}_{\bar{a}}$ and $\widetilde{\psi}_{\mu}$ have been defined in (4.9). Then the quadratic terms in the fermionic action, evaluated in the background (4.23), takes the form

$$
S_{f}=\frac{1}{2 \pi \alpha^{\prime}} \frac{Q_{0}}{P_{0}} \int d^{4} x \sqrt{\operatorname{det} G^{(0)}} \mathcal{L}_{f},
$$

where

$$
\begin{aligned}
\mathcal{L}_{f}= & -\frac{1}{2}\left[\sum_{r=7}^{28} \bar{\varphi}_{r}\left\{\Gamma^{\mu} D_{\mu}+\frac{1}{2 \sqrt{2}} \Gamma^{\rho \sigma}\left(\bar{F}_{\rho \sigma}^{1} \Gamma^{4}+\bar{F}_{\rho \sigma}^{2} \Gamma^{5}\right)\right\} \varphi_{r}\right. \\
& +\bar{\psi}_{\mu} \Gamma^{\mu \nu \rho} D_{\nu} \psi_{\rho}+\bar{\lambda} \Gamma^{\mu} D_{\mu} \lambda \\
& +\frac{1}{4 \sqrt{2}} \bar{\psi}_{\mu}\left[-\Gamma^{\mu \nu \rho \sigma}+2 g^{\mu \sigma} g^{\nu \rho}+2 \Gamma^{\mu \rho \nu} \Gamma^{\sigma}+\Gamma^{\mu \nu} \Gamma^{\rho \sigma}\right]\left(\bar{F}_{\rho \sigma}^{1} \Gamma^{4}+\bar{F}_{\rho \sigma}^{2} \Gamma^{5}\right) \psi_{\nu} \\
& \left.+\frac{1}{4}\left[\bar{\psi}_{\mu} \Gamma^{\rho \sigma} \Gamma^{\mu}\left(\bar{F}_{\rho \sigma}^{1} \Gamma^{4}+\bar{F}_{\rho \sigma}^{2} \Gamma^{5}\right) \lambda-\bar{\lambda}\left(\bar{F}_{\rho \sigma}^{1} \Gamma^{4}+\bar{F}_{\rho \sigma}^{2} \Gamma^{5}\right) \Gamma^{\mu} \Gamma^{\rho \sigma} \psi_{\mu}\right]\right] .
\end{aligned}
$$

To this we add the gauge fixing term

$$
\frac{1}{2 \pi \alpha^{\prime}} \frac{Q_{0}}{P_{0}} \int d^{4} x \sqrt{\operatorname{det} G^{(0)}} \mathcal{L}_{g f}^{\prime}
$$

where

$$
\mathcal{L}_{g f}^{\prime}=\frac{1}{4} \bar{\psi}_{\mu} \Gamma^{\mu} \Gamma^{\nu} D_{\nu} \Gamma^{\rho} \psi_{\rho}
$$

The structure of the ghost action can be determined as follows. Since the gauge fixing term is $\Gamma^{\mu} \psi_{\mu}$, the lagrangian density for the spinor valued bosonic ghost fields $\tilde{b}, \tilde{c}$ is proportional to $\overline{\tilde{b}} \Gamma^{\mu} \delta \psi_{\mu}$, where $\delta \psi_{\mu}$ is the variation of $\psi_{\mu}$ under the supersymmetry transformation with parameter $\tilde{c}$. Using the supersymmetry transformation laws of the ten dimensional fields given in (4.8), and the relations between the ten and the four dimensional fields given in (4.37), we find that in the near horizon background (4.23),

$$
\begin{aligned}
\delta \psi_{\mu} & =D_{\mu} \tilde{c}+\frac{1}{4 \sqrt{2}}\left(4 \delta_{\mu}^{\rho} \Gamma^{\sigma}-\Gamma_{\mu} \Gamma^{\rho \sigma}\right)\left(\bar{F}_{\rho \sigma}^{(1)} \Gamma^{4}+\bar{F}_{\rho \sigma}^{(2)} \Gamma^{5}\right) \tilde{c}+\cdots \\
\delta \lambda & =-\frac{1}{4} \Gamma^{\rho \sigma}\left(\bar{F}_{\rho \sigma}^{(1)} \Gamma^{4}+\bar{F}_{\rho \sigma}^{(2)} \Gamma^{5}\right) \tilde{c} .
\end{aligned}
$$

where $\cdots$ denotes terms which vanish in the background (4.23). Thus

$$
\Gamma^{\mu} \delta \psi_{\mu}=\Gamma^{\mu} D_{\mu} \tilde{c},
$$


and the ghost lagrangian density is proportional to

$$
\mathcal{L}_{f ; g h o s t} \propto \overline{\tilde{b}} \Gamma^{\mu} D_{\mu} \tilde{c} .
$$

Quantization of the gravitino also requires the introduction of a third spin $1 / 2$ bosonic ghost field. This comes from the special nature of the gauge fixing term given in (4.41); to get this term we first insert into the path integral the gauge fixing term $\delta\left(\Gamma^{\mu} \psi_{\mu}-\xi(x)\right)$ for some arbitrary space-time dependent spinor $f(x)$; and then average over all $\xi(x)$ with a weight factor of $\exp (-\bar{\xi} \not D \xi)$. The integration over $\xi$ introduces an extra factor of $\operatorname{det} \not D$ which needs to be canceled by an additional spin half bosonic ghost with the standard kinetic operator proportional to $\not D$. Denoting the new ghost field by $\tilde{e}$ we get the additional ghost action to be

$$
\mathcal{L}_{f ; g h o s t}^{\prime} \propto \overline{\tilde{e}} \Gamma^{\mu} D_{\mu} \tilde{e} .
$$

In the fermionic sector only the fields $\varphi_{r}, \bar{\varphi}_{r}$ are $\mathrm{SO}(22)$ vectors. Rest of the fields including all the ghosts are $\mathrm{SO}(22)$ singlets. As before we shall analyze the contribution to the one loop effective action from $\mathrm{SO}(22)$ vector fields only.

\section{Eigenvalues, heat kernel and one loop effective action in the matter sector}

In this section we shall compute the eigenvalues and eigenfunctions of the kinetic operator in the matter sector and use it to calculate the logarithmic correction to the extremal black hole entropy. The $\mathrm{SO}(22)$ symmetry guarantees that at the quadratic level there is no mixing between fields carrying different $r$ values, so we can analyze one $r$ value at a time.

We first focus on the bosonic fields. From the structure of the action we see that the fields $\phi_{a r}$ for $3 \leq a \leq 6$ do not enter $\mathcal{L}_{\text {flux }}$; so their heat kernel is given by the standard heat kernel of scalar fields computed with $\mathcal{L}_{\text {standard }}+\mathcal{L}_{g f}$. The field $\phi_{2 r}$ mixes with the component of $\mathcal{A}^{(r)}$ along $S^{2}$ and the field $\phi_{1 r}$ mixes with the component of $\mathcal{A}^{(r)}$ along $A d S_{2}$. Thus we can separately analyze these two cases. This reduces the problem to that of a mixing between a single scalar and a vector field.

First we shall consider the mixing between $\phi_{2 r}$ and the component of $\mathcal{A}^{(r)}$ along $S^{2}$. To avoid proliferation of indices we drop the $\mathrm{SO}(22)$ and $\mathrm{SO}(6)$ indices on the fields, define $g_{\alpha \beta} \equiv G_{\alpha \beta}^{(0)}$ and express the relevent quadratic term in the action as

$$
-\frac{1}{2} \int \sqrt{\operatorname{det} g}\left[\left(\phi \mathcal{A}_{\alpha}\right)\left(\begin{array}{cc}
-\square+2 a^{-2} & -2 a^{-1} \varepsilon^{\gamma \beta} D_{\gamma} \\
-2 a^{-1} \varepsilon^{\alpha \gamma} D_{\gamma}-g^{\alpha \beta} \square+R^{\alpha \beta}+D^{\alpha} D^{\beta}
\end{array}\right)\left(\begin{array}{c}
\phi \\
\mathcal{A}_{\beta}
\end{array}\right)-\mathcal{A}_{\alpha} D^{\alpha} D^{\beta} \mathcal{A}_{\beta}\right],
$$

up to an overall multiplicative factor. The last term is the gauge fixing term. In order to construct the heat kernel of the combined system of the scalar and the gauge fields we need to find the eigenstates of the kinetic operator appearing in (5.1). For this we first decompose the gauge field as

$$
\mathcal{A}_{\alpha}=D_{\alpha} \psi+\varepsilon_{\alpha}^{\beta} D_{\beta} \chi,
$$

where $\psi$ and $\chi$ are scalars on $S^{2}$. Substituting this into (5.1) we get

$$
\begin{aligned}
& -\frac{1}{2} \int \sqrt{\operatorname{det} g}\left[\left(\phi \varepsilon_{\alpha}^{\alpha \prime} D_{\alpha \prime} \chi\right)\left(\begin{array}{cc}
-\square+2 a^{-2} & -2 a^{-1} \varepsilon^{\gamma \beta} D_{\gamma} \\
-2 a^{-1} \varepsilon^{\alpha \gamma} D_{\gamma} & -g^{\alpha \beta} \square+R^{\alpha \beta}+D^{\alpha} D^{\beta}
\end{array}\right)\left(\begin{array}{c}
\phi \\
\varepsilon_{\beta}^{\beta \prime} D_{\beta \prime} \chi
\end{array}\right)\right. \\
& \left.\quad-D_{\alpha} \psi D^{\alpha} D^{\beta} D_{\beta} \psi\right] .
\end{aligned}
$$


Note that the part of $\mathcal{A}_{\alpha}$ involving $\psi$ does not contribute to the first term and the part of $\mathcal{A}_{\alpha}$ involving $\chi$ does not contribute to the second term. We now decompose $\phi, \psi$ and $\chi$ as

$$
\phi=\sum_{n} a_{n} f_{n}(x), \quad \chi=\sum_{n} \frac{1}{\sqrt{\kappa}_{n}} b_{n} f_{n}(x), \quad \psi=\sum_{n} \frac{1}{\sqrt{\kappa}_{n}} c_{n} f_{n}(x),
$$

where $\left\{f_{n}\right\}$ is an orthonormal basis of eigenfunctions of the scalar $-\square$ operator with eigenvalues $\left\{\kappa_{n}\right\}$. Substituting this into (5.3) we get

$$
-\frac{1}{2} \sum_{n}\left[\left(\begin{array}{ll}
a_{n} & b_{n}
\end{array}\right)\left(\begin{array}{cc}
\kappa_{n}+2 a^{-2} & -2 a^{-1} \sqrt{\kappa_{n}} \\
-2 a^{-1} \sqrt{\kappa_{n}} & \kappa_{n}
\end{array}\right)\left(\begin{array}{l}
a_{n} \\
b_{n}
\end{array}\right)+\kappa_{n} c_{n} c_{n}\right] .
$$

This shows that the eigenvalues of the modes labelled by $c_{n}$ are not affected by the mixing with the scalars. On the other hand the eigenvalues of the modes labelled by $a_{n}, b_{n}$ change from $\kappa_{n}$ to

$$
\kappa_{n}+a^{-2} \pm a^{-1} \sqrt{4 \kappa_{n}+a^{-2}},
$$

for $\kappa_{n}>0$. For $\kappa_{n}=l(l+1) a^{-2}$ with $l>0$ this gives the eigenvalues

$$
(l-1) l a^{-2}, \quad(l+1)(l+2) a^{-2} .
$$

Thus for each pair of modes with $l>0$, one has its $l$ value shifted by +1 and one has its $l$ value shifted by -1 . Finally for $l=0$ there are no modes from $b_{n}$, and the eigenvalue of the mode $a_{n}$ shifts from 0 in the absence of flux to $2 a^{-2}$. This effectively causes a shift of the $l=0$ eigenvalue to $l=1$ eigenvalue. Thus the net additional contribution to the trace of the heat kernel on $S^{2}$ from the scalar and the gauge field is given by

$$
\begin{aligned}
\delta K_{S^{2}}^{v+s}= & \frac{1}{4 \pi a^{2}}\left[\sum_{l=0}^{\infty}(2 l+1)\left\{e^{-s(l+1)(l+2) / a^{2}}-e^{-s l(l+1) / a^{2}}\right\}\right. \\
& \left.+\sum_{l=1}^{\infty}(2 l+1)\left\{e^{-s(l-1) l / a^{2}}-e^{-s l(l+1) / a^{2}}\right\}\right],
\end{aligned}
$$

where the $l=0$ term in the first sum takes into account the shift of the scalar mode with $l=0$ to $l=1$. We now break this as a sum of four different sums and shift $l \rightarrow l \mp 1$ in the first and the third terms. This gives

$$
\begin{aligned}
\delta K_{S^{2}}^{v+s}= & \frac{1}{4 \pi a^{2}}\left[\sum_{l=1}^{\infty}(2 l-1) e^{-s l(l+1) / a^{2}}-\sum_{l=0}^{\infty}(2 l+1) e^{-s l(l+1) / a^{2}}\right. \\
& \left.\quad+\sum_{l=0}^{\infty}(2 l+3) e^{-s l(l+1) / a^{2}}-\sum_{l=1}^{\infty}(2 l+1) e^{-s l(l+1) / a^{2}}\right] \\
= & \frac{1}{2 \pi a^{2}} .
\end{aligned}
$$

Thus the net contribution to the heat kernel from a vector on $S^{2}$ and the scalar on $S^{2}$ with which the vector mixes is given by

$$
K_{S^{2}}^{v+s}=K_{S^{2}}^{v}+K_{S^{2}}^{s}+\frac{1}{2 \pi a^{2}}=3 K_{S^{2}}^{s},
$$

where in the last step we have used (3.10).

A similar analysis can be done for the mixing between $\phi_{1 r}$ and the component of the vector field $\mathcal{A}^{(r)}$ along $A d S_{2}$. The main difference between the $S^{2}$ and the $A d S_{2}$ case is that for $A d S_{2}$ the mixing term (5.1) is replaced by

$$
-\frac{1}{2} \int \sqrt{\operatorname{det} g}\left[\left(\phi \mathcal{A}_{m}\right)\left(\begin{array}{cc}
-\square-2 a^{-2} & 2 i a^{-1} \varepsilon^{p n} D_{p} \\
2 i a^{-1} \varepsilon^{m p} D_{p}-g^{m n} \square+R^{m n}+D^{m} D^{n}
\end{array}\right)\left(\begin{array}{c}
\phi \\
\mathcal{A}_{n}
\end{array}\right)-\mathcal{A}_{m} D^{m} D^{n} \mathcal{A}_{n}\right] .
$$


One can now analyze its effect on the eigenvalues of the kinetic operator exactly as in the case of $S^{2}$. The final outcome of this analysis is that if we denote the eigenvalue of $(d \delta+\delta d)$ on a vector field of the form $\varepsilon_{n}^{p} D_{p} \chi$ or a scalar field on $A d S_{2}$ by $\kappa \equiv\left(\lambda^{2}+\frac{1}{4}\right) / a^{2}$, then acting on fields $\phi$ and $\mathcal{A}_{n}$ carrying this eigenvalue the kinetic operator coming from the first term takes the form:

$$
\left(\begin{array}{cc}
\kappa-2 a^{-2} & 2 i \sqrt{\kappa} a^{-1} \\
2 i \sqrt{\kappa} a^{-1} & \kappa
\end{array}\right)
$$

Although this matrix is complex, it is diagonalizable with a (complex) orthogonal matrix and gives eigenvalues

$$
a^{-2}\left[(\lambda \pm i)^{2}+\frac{1}{4}\right]
$$

Thus the mixing between the scalar and the vector on $A d S_{2}$ shifts the parameter $\lambda$ by $i$ for one set of states and $-i$ for another set of states. The kinetic operator in the second term of (5.11) continues to have the eigenvalue $\left(\lambda^{2}+\frac{1}{4}\right) / a^{2}$ on fields of the form $D_{n} \psi$. As a result the net change in the heat kernel of the scalar and the vector on $A d S_{2}$ is given by ${ }^{15}$

$$
\delta K_{A d S_{2}}^{v+s}=\frac{1}{2 \pi a^{2}} \exp [-\bar{s} / 4] \int_{0}^{\infty} d \lambda \lambda \tanh (\pi \lambda)\left[e^{-\bar{s}(\lambda+i)^{2}}+e^{-\bar{s}(\lambda-i)^{2}}-2 e^{-\bar{s} \lambda^{2}}\right] .
$$

We now shift the integration variable $\lambda \rightarrow \lambda \mp i$ in the first two terms and express this as

$$
\begin{aligned}
\delta K_{A d S_{2}}^{v+s}= & \frac{1}{2 \pi a^{2}} \exp [-\bar{s} / 4]\left[\int_{i}^{i+\infty} d \lambda(\lambda-i) \tanh (\pi \lambda-i \pi) e^{-\bar{s} \lambda^{2}}\right. \\
& \left.+\int_{-i}^{-i+\infty} d \lambda(\lambda+i) \tanh (\pi \lambda+i \pi) e^{-\bar{s} \lambda^{2}}-2 \int_{0}^{\infty} d \lambda \lambda \tanh (\pi \lambda) e^{-\bar{s} \lambda^{2}}\right] .
\end{aligned}
$$

Using $\tanh (x \pm i \pi)=\tanh x$ and the fact that the integrands in (5.15) do not have any poles for $\operatorname{Re}(\lambda)>0$, we can deform the integration contour in the first integral as a contour from $i$ to 0 lying in the $\operatorname{Re}(\lambda)>0$ region and a contour from 0 to $\infty$ along the real axis. Similarly the integration contour in the second integral can be deformed to a contour from $-i$ to 0 lying in the $\operatorname{Re}(\lambda)>0$ region and a contour from 0 to $\infty$ along the real axis. The total contribution from the contours from 0 to $\infty$ cancel the last term in (5.15), and we get

$$
\delta K_{A d S_{2}}^{v+s}=\frac{1}{2 \pi a^{2}} \exp [-\bar{s} / 4]\left[\int_{i}^{0(+)} d \lambda(\lambda-i) \tanh (\pi \lambda) e^{-\bar{s} \lambda^{2}}+\int_{-i}^{0(+)} d \lambda(\lambda+i) \tanh (\pi \lambda) e^{-\bar{s} \lambda^{2}}\right]
$$

where the superscript $(+)$ denotes that we are integrating along a contour in the $R e(\lambda)>0$ region. Making a change of variables $\lambda \rightarrow-\lambda$ in the second term we get

$$
\delta K_{A d S_{2}}^{v+s}=\frac{1}{2 \pi a^{2}} \exp [-\bar{s} / 4]\left[\int_{i}^{0(+)} d \lambda(\lambda-i) \tanh (\pi \lambda) e^{-\bar{s} \lambda^{2}}-\int_{i}^{0(-)} d \lambda(\lambda-i) \tanh (\pi \lambda) e^{-\bar{s} \lambda^{2}}\right]
$$

\footnotetext{
${ }^{15}$ Note that as $\lambda \rightarrow 0$ the integrand grows as $\exp (3 \bar{s} / 4)$. Thus the eigenvalues of the kinetic operator are negative and the path integral is not well defined, reflected in the fact that the integration over $s$ will diverge for large $s$ if we try to carry out the integration over $s$ first for a fixed $\lambda$. Physically this divergence is a consequence of the imaginary background electric field in the euclidean $A d S_{2}$ space. However as we shall see, if we carry out the integration over $\lambda$ first then there is a cancelation and the exponentially divergent contribution in the large $s$ limit is removed. This procedure is consistent with the rules for computing loop amplitudes in string theory, where the integration over the modular parameter (the analog of $s$ ) is carried out at the end, after we have integrated / summed over the eigenvalues of the kinetic operator. Presumably at the level of the path integral this corresponds to deforming the path integration contour to the complex configuration space where the path integral is well defined, as e.g. in [113]. Further justification of this procedure can be found in appendix A.
} 
We now have a closed clockwise contour. The result of this contour integral can be easily evaluated in terms of the residue at the pole at $\lambda=i / 2$, and we get

$$
\delta K_{A d S_{2}}^{v+s}=-\frac{1}{2 \pi a^{2}}
$$

Thus the net contribution to the trace of the heat kernel from a vector of $A d S_{2}$ and the scalar on $A d S_{2}$ with which the vector mixes is given by

$$
K_{A d S_{2}}^{v+s}=K_{A d S_{2}}^{v}+K_{A d S_{2}}^{s}-\frac{1}{2 \pi a^{2}}=3 K_{A d S_{2}}^{s}
$$

where in the last step we have used (3.12).

We can now use these results to compute the net contribution to the heat kernel from the bosonic fields of a matter multiplet on $A d S_{2} \times S^{2}$. First of all there are four scalars which do not mix with the vector; their contribution will be given by $4 K_{A d S_{2}}^{s} K_{S^{2}}^{s}$. Then we have a vector of $S^{2}$ which mixes with one of the remaining scalars, giving a contribution $K_{S^{2}}^{v+s} K_{A d S_{2}}^{s}$ with $K_{S^{2}}^{v+s}$ given in (5.10). Next we have a vector along $A d S_{2}$ that mixes with the remaining scalar and gives a contribution $K_{S^{2}}^{s} K_{A d S_{2}}^{v+s}$ with $K_{A d S_{2}}^{v+s}$ given in (5.19). Finally we have a pair of ghosts whose contribution $-2 K_{A d S_{2}}^{s} K_{S^{2}}^{s}$ needs to be added. Thus the net contribution from the six scalars and one vector of the matter multiplet is given by

$K_{A d S_{2} \times S^{2}}^{v+6 s}(0 ; s)=4 K_{A d S_{2}}^{s} K_{S^{2}}^{s}+K_{A d S_{2}}^{s} K_{S^{2}}^{v+s}+K_{S^{2}}^{s} K_{A d S_{2}}^{v+s}-2 K_{A d S_{2}}^{s} K_{S^{2}}^{s}=8 K_{A d S_{2}}^{s}(0 ; s) K_{S^{2}}^{s}(0 ; s)$

Note that the small $s$ expansion of this quantity (and a similar result for the trace of the fermionic heat kernel given in (5.30)) could be computed using the heat kernel expansion discussed e.g. in [87]. However (5.20) and (5.30) also has information about the large $s$ behaviour. This is needed to identify and subtract the zero mode contributions.

Let us now consider the effect of the background flux on the fermionic fields in the matter multiplet. These fields are the fields $\varphi_{r}$ appearing in (4.39), and transform in the vector representation of SO(22). From (4.23), (4.39), and the representations of the gamma matrices given in (4.6), we see that the Dirac operator acting on the fermions takes the form ${ }^{16}$

$$
\not{D}=\not_{S^{2}}+\sigma_{3} \mathcal{D}_{A d S_{2}}
$$

where

$$
\mathcal{D}_{S^{2}}=\not D_{S^{2}}-\frac{i}{2} a^{-1} \widehat{\Gamma}^{5} \tau_{3}, \quad \mathcal{D}_{A d S_{2}}=\not D_{A d S_{2}}-\frac{1}{2} a^{-1} \widehat{\Gamma}^{4}
$$

$\not D_{S^{2}}$ and $\not D_{A d S_{2}}$ have been defined in (3.26), (3.27), and $\widehat{\Gamma}^{4}$ and $\widehat{\Gamma}^{5}$ are two of the six SO(6) gamma matrices satisfying

$$
\left\{\widehat{\Gamma}^{i}, \widehat{\Gamma}^{j}\right\}=2 \delta_{i j}, \quad\left[\widehat{\Gamma}^{i}, \sigma_{a}\right]=0=\left[\widehat{\Gamma}^{i}, \tau_{a}\right], \quad 1 \leq a \leq 3, \quad 4 \leq i, j \leq 9 .
$$

\footnotetext{
${ }^{16}$ Although the original fermions are chiral - in the sense that their chirality property under the spacetime Lorentz group $\mathrm{SO}(4)$ is correlated with their chirality under the internal R-symmetry group $\mathrm{SO}(6)$ - in order to compute the eigenvalue of the Dirac operator we shall ignore the chirality projection and then take appropriate square root of the determinant. Since this doubles the number of fermionic degrees of freedom, the action is not manifestly supersymmetric. We can avoid this by appropriately pairing the fermions in the dimensionally reduced four dimensional theory to construct Dirac fermions without using any additional fermionic degrees of freedom. Thus in this description we can maintain manifest supersymmetry. This is essential if we make use of supersymmetry in evaluating the path integral; e.g. using localization arguments [69]. However for the explicit computation of the one loop determinant the loss of manifest supersymmetry of the action will not be a problem.
} 
One can easily check that $\not D_{S^{2}}$ and $\sigma_{3} \not \mathcal{D}_{A d S_{2}}$ anticommute. Hence $\not{D}^{2}=\mathcal{D}_{S^{2}}^{2}+\mathcal{D}_{A d S_{2}}^{2}$, the eigenvalues of $\not^{2}$ are given by the sum of the eigenvalues of $\not_{S^{2}}^{2}$ and $\not{D}_{A d S_{2}}^{2}$, and the trace of the heat kernel of $\not D$ is given by -1 times the product of the traces of the heat kernels of $\not_{S^{2}}$ and $\not D_{A d S_{2}}$. Thus we first need to find the eigenvalues of $\mathcal{D}_{S^{2}}$ and $\mathcal{D}_{A d S_{2}}$. Since $\widehat{\Gamma}^{5} \tau_{3}$ and $\widehat{\Gamma}^{4} \sigma_{3}$ each have eigenvalues \pm 1 and commute with $\not_{S^{2}}$ and $\not_{A d S_{2}}$ respectively, it follows from (5.22) that the eigenvalues of $\not_{S^{2}}$ are given by the eigenvalues of $\not D_{S^{2}} \pm i a^{-1} / 2$, and the eigenvalues of $\not_{A d S_{2}}$ are given by the eigenvalues of $\not D_{A d S_{2}} \pm a^{-1} / 2$, Using this result and eqs. (3.29), (3.35) we see that the eigenvalues of $\mathcal{D}_{S^{2}}$ are given by $\pm i a^{-1}\left(l+1 \pm \frac{1}{2}\right)$ and the eigenvalues of $\mathcal{D}_{A d S_{2}}$ are given by $\pm i a^{-1}\left(\lambda \pm \frac{i}{2}\right)$. As a result $K_{S^{2}}^{f}$ defined in $(3.33)$ changes to ${ }^{17}$

$$
K_{S^{2}}^{f \prime}(0 ; s)=-\frac{1}{4 \pi a^{2}} \sum_{l=0}^{\infty}(2 l+2)\left[e^{-s\left(l+\frac{3}{2}\right)^{2} / a^{2}}+e^{-s\left(l+\frac{1}{2}\right)^{2} / a^{2}}\right]
$$

and $K_{A d S_{2}}^{f}$ given in (3.36) is replaced by

$$
\begin{aligned}
K_{A d S_{2}}^{f \prime}(0 ; s)= & -\frac{1}{2 \pi a^{2}} \int_{0}^{\infty} d \lambda\left[e^{-\bar{s}\left(\lambda+\frac{i}{2}\right)^{2}}+e^{-\bar{s}\left(\lambda-\frac{i}{2}\right)^{2}}\right] \lambda \operatorname{coth}(\pi \lambda) \\
= & -\frac{1}{2 \pi a^{2}} \int_{i / 2}^{\frac{i}{2}+\infty} d \lambda e^{-s \lambda^{2} / a^{2}}\left(\lambda-\frac{i}{2}\right) \tanh (\pi \lambda) \\
& -\frac{1}{2 \pi a^{2}} \int_{-i / 2}^{-\frac{i}{2}+\infty} d \lambda e^{-s \lambda^{2} / a^{2}}\left(\lambda+\frac{i}{2}\right) \tanh (\pi \lambda),
\end{aligned}
$$

where in the second step we have shifted $\lambda \rightarrow \lambda-\frac{i}{2}$ in the first term and $\lambda \rightarrow \lambda+\frac{i}{2}$ in the second term.

Changing $l \rightarrow l-1$ in the first term in (5.24) and defining $\bar{s}=s / a^{2}$ we get

$$
K_{S^{2}}^{f \prime}(0 ; s)=-\frac{1}{4 \pi a^{2}} e^{-\bar{s} / 4} \sum_{l=0}^{\infty} e^{-\bar{s} l(l+1)}(2 l+2 l+2)=-\frac{1}{2 \pi a^{2}} e^{-\bar{s} / 4} \sum_{l=0}^{\infty} e^{-\bar{s} l(l+1)}(2 l+1) .
$$

On the other hand in (5.25) we deform the first integration contour to over the range $i / 2$ to 0 and 0 to $\infty$ and the second integration contour to over the range $-i / 2$ to 0 and 0 to $\infty$. This gives

$$
\begin{aligned}
K_{A d S_{2}}^{f \prime}(0 ; s)= & -\frac{1}{2 \pi a^{2}} \int_{i / 2}^{0(+)} d \lambda e^{-s \lambda^{2} / a^{2}}\left(\lambda-\frac{i}{2}\right) \tanh (\pi \lambda) \\
& -\frac{1}{2 \pi a^{2}} \int_{-i / 2}^{0(+)} d \lambda e^{-s \lambda^{2} / a^{2}}\left(\lambda+\frac{i}{2}\right) \tanh (\pi \lambda) \\
& -\frac{1}{\pi a^{2}} \int_{0}^{\infty} d \lambda e^{-s \lambda^{2} / a^{2}} \lambda \tanh (\pi \lambda) .
\end{aligned}
$$

As before the superscript $(+)$ denotes that the contour lies in the $\operatorname{Re}(\lambda)>0$ region. Changing $\lambda \rightarrow-\lambda$ in the second integral gives

$$
\begin{aligned}
K_{A d S_{2}}^{f \prime}(0 ; s)= & -\frac{1}{2 \pi a^{2}} \int_{i / 2}^{0(+)} d \lambda e^{-s \lambda^{2} / a^{2}}\left(\lambda-\frac{i}{2}\right) \tanh (\pi \lambda) \\
& +\frac{1}{2 \pi a^{2}} \int_{i / 2}^{0(-)} d \lambda e^{-s \lambda^{2} / a^{2}}\left(\lambda-\frac{i}{2}\right) \tanh (\pi \lambda) \\
& -\frac{1}{\pi a^{2}} \int_{0}^{\infty} d \lambda e^{-s \lambda^{2} / a^{2}} \lambda \tanh (\pi \lambda) .
\end{aligned}
$$

\footnotetext{
${ }^{17}$ We are giving the result for the heat kernel per Dirac fermion.
} 
We now note that the first and second integrals can be combined into a singe clockwise contour and the result vanishes since the integrand does not have any singularity enclosed by the contour. Thus we have

$$
K_{A d S_{2}}^{f \prime}(0 ; s)=-\frac{1}{\pi a^{2}} \int_{0}^{\infty} d \lambda e^{-s \lambda^{2} / a^{2}} \lambda \tanh (\pi \lambda) .
$$

Combining this with (5.26) we get the net contribution to the effective heat kernel of a Dirac fermion on $A d S_{2} \times S^{2}$ in the presence of background flux:

$$
\begin{aligned}
K^{f \prime}(0 ; s) & =-K_{A d S_{2}}^{f \prime}(0 ; s) K_{S^{2}}^{f \prime}(0 ; s) \\
& =-\frac{1}{2 \pi^{2} a^{4}} e^{-\bar{s} / 4} \sum_{l=0}^{\infty} e^{-\bar{s} l(l+1)}(2 l+1) \int_{0}^{\infty} d \lambda e^{-\bar{s} \lambda^{2}} \lambda \tanh (\pi \lambda) \\
& =-4 K^{s}(0 ; s),
\end{aligned}
$$

where $K^{s}(0 ; s)=K_{S^{2}}^{s}(0 ; s) K_{A d S_{2}}^{s}(0 ; s)$ is the heat kernel of a scalar on $A d S_{2} \times S^{2}$ as given in (2.12). Since a single matter multiplet contains four Weyl fermions, or equivalently two Dirac fermions, the net contribution to the heat kernel from the fermions is given by $-8 K^{s}(0 ; s)$. This exactly cancels the contribution (5.20), showing that the net contribution to the heat kernel from a matter multiplet is zero.

Our result also shows that no subtraction of the type described in (3.15) is needed to regulate the infrared divergences. Mathematically it is a consequence of an additional $s$ independent constant term that arose in the expression for $\delta K_{A d S_{2}}^{v+s} K_{S^{2}}^{s}$. However physically this is somewhat surprising given that the subtraction was needed to remove the contribution from the zero modes of the vector fields on $A d S_{2} \times S^{2}$. In appendix A we have provided a justification of this procedure by carefully analyzing the contribution from integration over these zero modes. We also note that since these zero modes transform non-trivially under a simultaneous rotation in $A d S_{2}$ and $S^{2}$, the argument of [69] shows that the contribution to the path integral due to these zero modes will cancel a similar contribution from the fermion zero modes. Put another way, supersymmetry allows us add a term to the action which does not change the result of the path integral but lifts the zero modes. Thus it appears that the analytic continuation procedure we have adopted, namely doing the $\lambda$ integral first and then the $s$ integral, automatically accounts for this cancelation. This clearly deserves further study.

This concludes our analysis leading to the result that the matter multiplet fields of $\mathcal{N}=4$ supergravity do not give any logarithmic correction to the entropy of a quarter BPS black holes. In fact since the heat kernel vanishes for all $s$, the full one loop contribution from the massless matter multiplet vanishes. ${ }^{18}$ Since we have not computed the contribution due to the gravity multiplet fields, our analysis does not produce the complete logarithmic correction to the entropy. Nevertheless our result has non-trivial prediction for the entropy. For this recall that there is a whole class of $\mathcal{N}=4$ supersymmetric string theories with different number of matter multiplet fields $[114,115]$. In these theories the quadratic action of fluctuating fields around the attractor geometry will have exactly the same structure as discussed here except that the index $r$ now runs over a lower number of values than 22. Since the quadratic action of the gravity multiplet fields is common to all these theories, the one loop contribution from these fields to the entropy will also be identical. The vanishing of the contribution from the matter sector then implies that for all the $\mathcal{N}=4$ supersymmetric theories the one loop contributions to the black hole entropy from the masless fields are identical. In particular the logarithmic corrections to the entropy — which we have argued earlier come only from the one loop contribution due to the massless fields — must also

\footnotetext{
${ }^{18}$ We do not rule out the possibility of a finite left-over contribution due to different ultra-violet cut-off on different terms imposed by string theory.
} 
be identical. This is consistent with the microscopic result for the entropy of quarter BPS states in a variety of $\mathcal{N}=4$ supersymmetric string theories. None of these theories have any logarithmic correction to the entropy of quarter BPS black holes irrespective of the number of matter multiplets they have $[12,13,15]$.

\section{Discussion}

In this paper we have analyzed the eigenvalues and eigenfunctions associated with the fluctuations of massless matter multiplet fields in the near horizon geometry of quarter BPS black holes in $\mathcal{N}=4$ supersymmetric string theories. This allows us to calculate the one loop effective action and the logarithmic correction to the Bekenstein-Hawking entropy due to the fields in the matter multiplet. We find that even though individual fields contribute to the effective action and logarithmic correction to the black hole entropy, the net contribution from all the fields in the matter multiplet vanishes. This is consistent with the fact that there are no logarithmic corrections to the microscopic entropy in $\mathcal{N}=4$ supersymmetric string theories. In particular since the logarithmic contribution to the microscopic entropy vanishes independent of how many matter multiplet fields we have in the theory, we would have run into an inconsistency if there had been a non-vanishing logarithmic contribution from the matter multiplet fields to the macroscopic entropy.

Ref. [116] presented a general analysis, based on the computation of the trace anomaly, which showed that the trace anomaly vanishes for on-shell backgrounds in $\mathcal{N}=4$ and $\mathcal{N}=8$ gauged supergravity theories. Since the trace anomaly is related to the coefficient of the $s$ independent term in the expansion of $K(0 ; s)$ via relations of the type described in (2.23), our result may appear to be similar in spirit to those in [116]. However the analysis of [116], being a local analysis, does not take into account the possible subtraction term given in (3.15) for removing the zero modes. Indeed, the results of [116] would change if we had replaced some of the fields by their dual description, e.g. the scalars by 2 -form fields. Our analysis shows the vanishing of $K(0 ; s)$ for all $s$ and hence also the regulated $\widehat{K}(0 ; s)$ given in $(3.15)$. Since $\widehat{K}(0 ; s)$ remains unchanged when we replace a field by its dual description, the vanishing of $\widehat{K}(0 ; s)$ holds irrespective of the duality frame we use to describe the fields.

One might naively conclude that the cancelation we have found is a result of supersymmetry. However examining the microscopic results for the black hole entropy we find that while quarter BPS black holes in $\mathcal{N}=4$ supersymmetric string theories have no logarithmic corrections to their entropy, $1 / 8$ BPS black holes in $\mathcal{N}=8$ supersymmetric string theories, having the same amount of supersymmetry as the quarter BPS black holes in $\mathcal{N}=4$ supersymmetric string theories, do have logarithmic corrections to their entropy. Thus the cancelation observed above cannot merely be a consequence of supersymmetry. Nevertheless the vanishing of the matter multiplet contribution to the logarithmic corrections is crucial for correct matching with the microscopic entropy of quarter BPS black holes in $\mathcal{N}=4$ supersymetric string theories, which do not have any logarithmic terms which depend on the number of matter multiplets.

It is clearly desirable to extend the computation to include the fields in the gravity multiplet, both in the $\mathcal{N}=4$ and $\mathcal{N}=8$ supersymmetric string theories, and verify that the macroscopic results are in agreement with the microscopic results given in (1.1). This can be done either by the brute force approach of diagonalizing the fluctuations in the gravity multiplet fields in the near horizon geometry, or possibly by carrying out a direct string one loop calculation as in [117]. The latter computation will give the complete one loop contribution, including the order one contribution from the massive states, in one step. In this case the answer would be given by an integration over the modular parameter $\tau$ of the torus, with its imaginary part playing the role of the integration variable $s$ and the integrand a generalized version of $K(0 ; s)$ that also takes into account the 
contribution from the massive modes. The difficulty in carrying out this program lies in the fact that we have to solve string theory in Ramond-Ramond background and then carry out an exact one loop calculation in this background. While this is not an easy task, it will be interesting to see if the pure spinor formalism [118] or the hybrid formalism of [119, 120] can be of help. An attempt to do this path integral using localization principle and semi-classical method can be found in [68].

\section{Acknowledgments}

We would like to thank Nabamita Banerjee, Atish Dabholkar, Justin David, Joao Gomes, Rajesh Gopakumar, Sameer Murthy and Boris Pioline for useful discussions. The work of A.S. was supported in part by the J. C. Bose fellowship of the Department of Science and Technology, India and by the Chaires Internationales de Recherche Blaise Pascal, France. S.B. would like to acknowledge IIT, Chennai and Center For High Energy Physics, IISc Bangalore, where some computations related to this work was performed.

\section{A Analysis of the zero mode contribution}

In the analysis of section 3 we had removed the zero mode contribution while evaluating the determinant of the kinetic operator for various fields. In this section we shall analyze the result of the zero mode integrals for the vector fields on $A d S_{2} \times S^{2}$ - the only fields containing zero modes which appeared in the explicit analysis of section 5 - and show that their contribution cancels against another contribution that was left out in the analysis of section 5 .

Let $A_{\mu}$ be a vector field of $A d S_{2} \times S^{2}$ and $g_{\mu \nu}$ be the background metric. This has the form

$$
g_{\mu \nu}=a^{2} g_{\mu \nu}^{(0)},
$$

where $a$ is the size parameter of $S^{2}$ and $A d S_{2}$ and $g_{\mu \nu}^{(0)}$ is independent of $a$. Now in our analysis in section 3 we have assumed that the integration over $A_{\mu}$ gives the determinant of the kinetic operator $(d \delta+\delta d)$ constructed from the metric $g_{\mu \nu}$. For this we need to normalize the path integral over $A_{\mu}$ such that

$$
\int\left[D A_{\mu}\right] \exp \left[-\int d^{4} x \sqrt{\operatorname{det} g} g^{\mu \nu} A_{\mu} A_{\nu}\right]=1 .
$$

Using (A.1) this may be expressed as

$$
\int\left[D A_{\mu}\right] \exp \left[-a^{2} \int d^{4} x \sqrt{\operatorname{det} g^{(0)}} g^{(0) \mu \nu} A_{\mu} A_{\nu}\right]=1 .
$$

From this we see that up to an $a$ independent normalization constant, $\left[D A_{\mu}\right]$ actually corresponds to integration with measure $\prod_{x} d\left(a A_{\mu}(x)\right)$. This in turn implies that integration over every zero mode of $A_{\mu}(x)$ with the measure induced from $\left[D A_{\mu}\right]$ will produce a factor of $a$.

Now for a non-zero mode, the path integral weighted by the exponential of the action produces a factor of $\kappa_{n}^{-1 / 2}$ where $\kappa_{n}$ is the eigenvalue of the kinetic operator. Since $\kappa_{n}$ has the form $b_{n} / a^{2}$ where $b_{n}$ is an $a$ independent constant, integration over a non-zero mode produces a factor proportional to $a$. Thus when we remove the contribution due to the zero modes, we remove a factor of $a$ for each zero mode. However the analysis of the previous paragraph showed that integration over the zero modes gives us back a factor of $a$. Thus the net result is that for computing the coefficient of the $\ln a$ term we can effectively ignore the subtraction described in (3.15) and continue to use the full heat kernel $K^{v}(0 ; s)$ provided we use the prescription that the $\ln$ a terms arise from integration over $s$ in the range $1 \ll s \ll a^{2}$ even though the integral $\int d s s^{-1} K^{v}(0 ; s)$ does not converge at large $s$. 
Looking back at our final expression (5.20) for the net contribution to the trace of the heat kernel from a matter multiplet, we see that the right hand side of this expression in fact vanishes rapidly as $s \rightarrow \infty$ since it is proportional to the scalar heat kernel in $A d S_{2}$ which does not have any zero mode contribution. So indeed we did not need to explicitly carry out any subtraction of the type given in (3.15). Technically this was due to the fact that there was another term that approached a constant as $s \rightarrow \infty$ and canceled the constant term in the trace of the vector heat kernel in $A d S_{2} \times S^{2}$. This new term arose from the product of $K_{S^{2}}^{s}$ and $\delta K_{A d S_{2}}^{v+s}$ given in (5.18). So if we can argue that the correct prescription for evaluating the contribution from $K_{S^{2}}^{s} \delta K_{A d S_{2}}^{v+s}$ to the $\ln a$ terms is to not subtract the constant term as $s \rightarrow \infty$, and restrict the integration over $s$ to the range $1 \ll s \ll a^{2}$, then our final result (5.20) will be justified; we do not subtract any constant either from the original $K_{A d S_{2} \times S^{2}}^{v}$, nor from the correction term $K_{S^{2}}^{s} \delta K_{A d S_{2}}^{v+s}$.

Thus our task now is to justify (5.18) for calculating the effect of the flux in $A d S_{2}$ without any subtraction. If we adopt this prescription then the net change proportional to $\ln a$ in $-\frac{1}{2} \ln \operatorname{det}(d \delta+$ $\delta d)$ due to the presence of the flux through $A d S_{2}$ will be given by

$$
\frac{1}{2} \int d^{4} x \sqrt{\operatorname{det} g} \int_{1 \ll s \ll a^{2}} \frac{d s}{s} K_{S^{2}}^{s}(0 ; s) \delta K_{A d S_{2}}^{v+s}(0 ; s) .
$$

If we denote by $u$ and $v$ the coordinates of $A d S_{2}$ and $S^{2}$ respectively, and pick a particular eigenfunction on $S^{2}$ with eigenvalue $c / a^{2}$ and eigenfunction $f(v)$, then the contribution from this eigenfunction on $S^{2}$ to (A.4) will be given by

$$
\frac{1}{2} \int d^{4} x|f(v)|^{2} \sqrt{\operatorname{det} g} \int_{1 \ll s \ll a^{2}} \frac{d s}{s} e^{-c s / a^{2}} \delta K_{A d S_{2}}^{v+s}(0 ; s)=-\frac{1}{4 \pi a^{2}} \ln \left(a^{2}\right) \int d^{4} x|f(v)|^{2} \sqrt{\operatorname{det} g} .
$$

Note that we have used the ad hoc prescription of restricting the integration range to $1 \ll s \ll a^{2}$; without this the integral will diverge from the large $s$ region for $c=0$. Now we shall verify the correctness of this result using an independent procedure that does not require this ad hoc prescription. For this we go back to (5.14). From this equation it is clear that the effect of the flux is to take a pair of eigenvalues $\left(c+\lambda^{2}+\frac{1}{4}\right) / a^{2}$ of $-\square_{S^{2}}-\square_{A d S_{2}}$ and shift them to $\left(c+(\lambda \pm i)^{2}+\frac{1}{4}\right) / a^{2}$. Now since we are interested in computing the determinant, we could also interpret this as shifting a factor of $\left(c+\lambda^{2}+\frac{1}{4}\right)^{2} / a^{4}$ in the determinant to $\left|c+(\lambda+i)^{2}+\frac{1}{4}\right|^{2} / a^{4}$. Thus the change in $-\frac{1}{2} \ln \operatorname{det}(d \delta+\delta d)$ can be written as

$$
\begin{aligned}
& \frac{1}{4 \pi a^{2}} \int_{\tilde{\epsilon}}^{\infty} \frac{d t}{t} \int d^{4} x|f(v)|^{2} \sqrt{\operatorname{det} g} \int_{0}^{\infty} d \lambda \lambda \tanh (\pi \lambda) \\
& {\left[\exp \left(-t\left|c+(\lambda+i)^{2}+\frac{1}{4}\right|^{2} / a^{4}\right)-\exp \left(-t\left(c+\lambda^{2}+\frac{1}{4}\right)^{2} / a^{4}\right)\right],}
\end{aligned}
$$

where we have used the fact that the distribution function of the parameter $\lambda$ is given by $\lambda \tanh (\pi \lambda) /\left(2 \pi a^{2}\right) . \quad \tilde{\epsilon}$ is an untraviolet cut-off of order 1 . This integral is manifestly convergent at large $t$ even for $c=0$ and does not have the problem mentioned in footnote 15. Since the integrand falls off rapidly for $t \gg a^{4}$, the possible $\ln a$ terms come from integration over the range $1 \ll t \ll a^{4}$. Using the method described in section 2 one can estimate the behaviour of the integrand in this range after carrying out the $\lambda$ integral, and finds the result:

$$
-\frac{1}{8 \pi a^{2}} \int d^{4} x|f(v)|^{2} \sqrt{\operatorname{det} g} \int_{1 \ll t \ll a^{4}} \frac{d t}{t} \simeq-\frac{1}{4 \pi a^{2}} \ln \left(a^{2}\right) \int d^{4} x|f(v)|^{2} \sqrt{\operatorname{det} g} .
$$

This is in perfect agreement with (A.5), showing that the prescription of using the full result $\delta K_{A d S_{2}}^{v+s}$ given in (5.18) without any subtraction and restricting the integration in the range $1 \ll s \ll a^{2}$ gives 
the correct $\ln a$ factors in the determinant. Of course since the final result (5.20) falls off sufficient rapidly for $s \rightarrow \infty$ we can drop the requirement of restricting the integration to the range $s \ll a^{2}$.

This concludes our proof that even after taking into account the possible additional factors of $\ln a$ which could arise from zero mode integration, (5.20) can be used to calculate the logarithmic correction to the black hole entropy due to the bosonic fields in the matter multiplet.

Open Access. This article is distributed under the terms of the Creative Commons Attribution Noncommercial License which permits any noncommercial use, distribution, and reproduction in any medium, provided the original author(s) and source are credited.

\section{References}

[1] R.M. Wald, Black hole entropy is the Noether charge, Phys. Rev. D 48 (1993) 3427 [gr-qc/9307038] [SPIRES].

[2] T. Jacobson, G. Kang and R.C. Myers, On black hole entropy, Phys. Rev. D 49 (1994) 6587 [gr-qc/9312023] [SPIRES].

[3] V. Iyer and R.M. Wald, Some properties of Noether charge and a proposal for dynamical black hole entropy, Phys. Rev. D 50 (1994) 846 [gr-qc/9403028] [SPIRES].

[4] T. Jacobson, G. Kang and R.C. Myers, Black hole entropy in higher curvature gravity, gr-qc/9502009 [SPIRES].

[5] A. Sen, Black Hole Entropy Function and the Attractor Mechanism in Higher Derivative Gravity, JHEP 09 (2005) 038 [hep-th/0506177] [SPIRES].

[6] A. Sen, Entropy function for heterotic black holes, JHEP 03 (2006) 008 [hep-th/0508042] [SPIRES].

[7] S. Ferrara, R. Kallosh and A. Strominger, N=2 extremal black holes, Phys. Rev. D 52 (1995) 5412 [hep-th/9508072] [SPIRES].

[8] A. Strominger, Macroscopic Entropy of $N=2$ Extremal Black Holes, Phys. Lett. B 383 (1996) 39 [hep-th/9602111] [SPIRES].

[9] S. Ferrara and R. Kallosh, Supersymmetry and Attractors, Phys. Rev. D 54 (1996) 1514 [hep-th/9602136] [SPIRES].

[10] A. Sen, Arithmetic of Quantum Entropy Function, JHEP 08 (2009) 068 [arXiv:0903.1477] [SPIRES].

[11] A. Dabholkar, J. Gomes, S. Murthy and A. Sen, to appear.

[12] G. Lopes Cardoso, B. de Wit, J. Kappeli and T. Mohaupt, Asymptotic degeneracy of dyonic $N=4$ string states and black hole entropy, JHEP 12 (2004) 075 [hep-th/0412287] [SPIRES].

[13] D.P. Jatkar and A. Sen, Dyon spectrum in CHL models, JHEP 04 (2006) 018 [hep-th/0510147] [SPIRES].

[14] J.R. David and A. Sen, CHL dyons and statistical entropy function from D1 - D5 system, JHEP 11 (2006) 072 [hep-th/0605210] [SPIRES].

[15] J.R. David, D.P. Jatkar and A. Sen, Dyon spectrum in generic $N=4$ supersymmetric $Z(N)$ orbifolds, JHEP 01 (2007) 016 [hep-th/0609109] [SPIRES].

[16] R. Dijkgraaf, E.P. Verlinde and H.L. Verlinde, Counting Dyons in $N=4$ String Theory, Nucl. Phys. B 484 (1997) 543 [hep-th/9607026] [SPIRES]. 
[17] D. Shih, A. Strominger and X. Yin, Recounting dyons in $N=4$ string theory, JHEP 10 (2006) 087 [hep-th/0505094] [SPIRES].

[18] D. Gaiotto, Re-recounting dyons in $N=4$ string theory, hep-th/0506249 [SPIRES].

[19] D. Shih and X. Yin, Exact Black Hole Degeneracies and the Topological String, JHEP 04 (2006) 034 [hep-th/0508174] [SPIRES].

[20] J.R. David, D.P. Jatkar and A. Sen, Product representation of dyon partition function in CHL models, JHEP 06 (2006) 064 [hep-th/0602254] [SPIRES].

[21] A. Dabholkar and S. Nampuri, Spectrum of Dyons and Black Holes in CHL orbifolds using Borcherds Lift, JHEP 11 (2007) 077 [hep-th/0603066] [SPIRES].

[22] J.R. David, D.P. Jatkar and A. Sen, Dyon spectrum in $N=4$ supersymmetric type-II string theories, JHEP 11 (2006) 073 [hep-th/0607155] [SPIRES].

[23] A. Dabholkar and D. Gaiotto, Spectrum of CHL dyons from genus-two partition function, JHEP 12 (2007) 087 [hep-th/0612011] [SPIRES].

[24] A. Sen, Walls of Marginal Stability and Dyon Spectrum in $N=4$ Supersymmetric String Theories, JHEP 05 (2007) 039 [hep-th/0702141] [SPIRES].

[25] A. Dabholkar, D. Gaiotto and S. Nampuri, Comments on the spectrum of CHL dyons, JHEP 01 (2008) 023 [hep-th/0702150] [SPIRES].

[26] N. Banerjee, D.P. Jatkar and A. Sen, Adding charges to $N=4$ dyons, JHEP 07 (2007) 024 [arXiv: 0705.1433] [SPIRES].

[27] A. Sen, Two Centered Black Holes and $N=4$ Dyon Spectrum, JHEP 09 (2007) 045 [arXiv: 0705.3874] [SPIRES].

[28] M.C.N. Cheng and E. Verlinde, Dying Dyons Don't Count, JHEP 09 (2007) 070 [arXiv:0706.2363] [SPIRES].

[29] A. Sen, Black Hole Entropy Function, Attractors and Precision Counting of Microstates, Gen. Rel. Grav. 40 (2008) 2249 [arXiv:0708.1270] [SPIRES].

[30] S. Banerjee, A. Sen and Y.K. Srivastava, Generalities of Quarter BPS Dyon Partition Function and Dyons of Torsion Two, JHEP 05 (2008) 101 [arXiv:0802.0544] [SPIRES].

[31] S. Banerjee, A. Sen and Y.K. Srivastava, Partition Functions of Torsion $>1$ Dyons in Heterotic String Theory on $T^{6}$, JHEP 05 (2008) 098 [arXiv:0802.1556] [SPIRES].

[32] A. Dabholkar, J. Gomes and S. Murthy, Counting all dyons in $N=4$ string theory, arXiv:0803.2692 [SPIRES].

[33] M.C.N. Cheng and E.P. Verlinde, Wall Crossing, Discrete Attractor Flow and Borcherds Algebra, SIGMA 4 (2008) 068 [arXiv:0806.2337] [SPIRES].

[34] S. Govindarajan and K. Gopala Krishna, Generalized Kac-Moody Algebras from CHL dyons, JHEP 04 (2009) 032 [arXiv:0807.4451] [SPIRES].

[35] M.C.N. Cheng and A. Dabholkar, Borcherds-Kac-Moody Symmetry of $N=4$ Dyons, Commun. Num. Theor. Phys. 3 (2009) 59 [arXiv:0809.4258] [SPIRES].

[36] S. Banerjee, A. Sen and Y.K. Srivastava, Genus Two Surface and Quarter BPS Dyons: The Contour Prescription, JHEP 03 (2009) 151 [arXiv:0808.1746] [SPIRES].

[37] M.C.N. Cheng and L. Hollands, A Geometric Derivation of the Dyon Wall-Crossing Group, JHEP 04 (2009) 067 [arXiv:0901.1758] [SPIRES]. 
[38] S. Govindarajan and K. Gopala Krishna, BKM Lie superalgebras from dyon spectra in $Z_{N}$ CHL orbifolds for composite N, JHEP 05 (2010) 014 [arXiv:0907.1410] [SPIRES].

[39] A. Dabholkar and J. Gomes, Perturbative tests of non-perturbative counting, JHEP 03 (2010) 128 [arXiv:0911.0586] [SPIRES].

[40] A. Sen, A Twist in the Dyon Partition Function, JHEP 05 (2010) 028 [arXiv:0911.1563] [SPIRES].

[41] A. Sen, Discrete Information from CHL Black Holes, JHEP 11 (2010) 138 [arXiv: 1002 .3857] [SPIRES].

[42] S. Govindarajan, BKM Lie superalgebras from twisted CHL dyons, to appear.

[43] M. Cvetič and D. Youm, Dyonic BPS saturated black holes of heterotic string on a six torus, Phys. Rev. D 53 (1996) 584 [hep-th/9507090] [SPIRES].

[44] M. Cvetič and A.A. Tseytlin, Solitonic strings and BPS saturated dyonic black holes, Phys. Rev. D 53 (1996) 5619 [Erratum ibid. D 55 (1997) 3907] [hep-th/9512031] [SPIRES].

[45] J.M. Maldacena, A. Strominger and E. Witten, Black hole entropy in M-theory, JHEP 12 (1997) 002 [hep-th/9711053] [SPIRES].

[46] G. Lopes Cardoso, B. de Wit and T. Mohaupt, Corrections to macroscopic supersymmetric black-hole entropy, Phys. Lett. B 451 (1999) 309 [hep-th/9812082] [SPIRES].

[47] G. Lopes Cardoso, B. de Wit and T. Mohaupt, Macroscopic entropy formulae and non-holomorphic corrections for supersymmetric black holes, Nucl. Phys. B 567 (2000) 87 [hep-th/9906094] [SPIRES].

[48] T. Mohaupt, Black hole entropy, special geometry and strings, Fortsch. Phys. 49 (2001) 3 [hep-th/0007195] [SPIRES].

[49] R. Aros, D.E. Diaz and A. Montecinos, Logarithmic correction to BH entropy as Noether charge, JHEP 07 (2010) 012 [arXiv: 1003.1083] [SPIRES].

[50] A. Sen, Quantum Entropy Function from $A d S_{2} / C F T_{1}$ Correspondence, Int. J. Mod. Phys. A 24 (2009) 4225 [arXiv:0809.3304] [SPIRES].

[51] J.M. Maldacena, Wilson loops in large-N field theories, Phys. Rev. Lett. 80 (1998) 4859 [hep-th/9803002] [SPIRES].

[52] S.-J. Rey and J.-T. Yee, Macroscopic strings as heavy quarks in large- $N$ gauge theory and anti-de Sitter supergravity, Eur. Phys. J. C 22 (2001) 379 [hep-th/9803001] [SPIRES].

[53] J. Gomis, T. Okuda and D. Trancanelli, Quantum 't Hooft operators and S-duality in $N=4$ super Yang-Mills, Adv. Theor. Math. Phys. 13 (2009) 1941 [arXiv:0904.4486] [SPIRES].

[54] N. Banerjee, D.P. Jatkar and A. Sen, Asymptotic Expansion of the $N=4$ Dyon Degeneracy, JHEP 05 (2009) 121 [arXiv:0810.3472] [SPIRES].

[55] S. Murthy and B. Pioline, A Farey tale for $N=4$ dyons, JHEP 09 (2009) 022 [arXiv:0904.4253] [SPIRES].

[56] A. Sen, Arithmetic of $N=8$ Black Holes, JHEP 02 (2010) 090 [arXiv:0908.0039] [SPIRES].

[57] J.J. Duistermaat and G.J. Heckman, On The Variation In The Cohomology Of The Symplectic Form Of The Reduced Phase Space, Invent. Math. 69 (1982) 259 [SPIRES].

[58] E. Witten, Topological Quantum Field Theory, Commun. Math. Phys. 117 (1988) 353 [SPIRES]. 
[59] E. Witten, The $N$ matrix model and gauged WZW models, Nucl. Phys. B 371 (1992) 191 [SPIRES].

[60] E. Witten, Mirror manifolds and topological field theory, hep-th/9112056 [SPIRES].

[61] E. Witten, Two-dimensional gauge theories revisited, J. Geom. Phys. 9 (1992) 303 [hep-th/9204083] [SPIRES].

[62] A.S. Schwarz and O. Zaboronsky, Supersymmetry and localization, Commun. Math. Phys. 183 (1997) 463 [hep-th/9511112] [SPIRES].

[63] O.V. Zaboronsky, Dimensional reduction in supersymmetric field theories, J. Phys. A 35 (2002) 5511 [SPIRES].

[64] N.A. Nekrasov, Seiberg-Witten Prepotential From Instanton Counting, Adv. Theor. Math. Phys. 7 (2004) 831 [hep-th/0206161] [SPIRES].

[65] V. Pestun, Localization of gauge theory on a four-sphere and supersymmetric Wilson loops, arXiv:0712.2824 [SPIRES].

[66] M.F. Atiyah, Elliptic operators and compact groups, Springer-Verlag, Berlin (1974).

[67] P. Shanahan, The atiyah-singer index theorem: An introduction, Springer-Verlag.

[68] C. Beasley et al., Why $Z_{B H}=\left|Z_{\text {top }}\right|^{2}$, hep-th/0608021 [SPIRES].

[69] N. Banerjee, S. Banerjee, R.K. Gupta, I. Mandal and A. Sen, Supersymmetry, Localization and Quantum Entropy Function, JHEP 02 (2010) 091 [arXiv: 0905.2686] [SPIRES].

[70] R.K. Kaul and P. Majumdar, Logarithmic correction to the Bekenstein-Hawking entropy, Phys. Rev. Lett. 84 (2000) 5255 [gr-qc/0002040] [SPIRES].

[71] S. Carlip, Logarithmic corrections to black hole entropy from the Cardy formula, Class. Quant. Grav. 17 (2000) 4175 [gr-qc/0005017] [SPIRES].

[72] T.R. Govindarajan, R.K. Kaul and V. Suneeta, Logarithmic correction to the Bekenstein-Hawking entropy of the BTZ black hole, Class. Quant. Grav. 18 (2001) 2877 [gr-qc/0104010] [SPIRES].

[73] A. Gregori et al., $R^{2}$ corrections and non-perturbative dualities of $N=4$ string ground states, Nucl. Phys. B 510 (1998) 423 [hep-th/9708062] [SPIRES].

[74] E. Kiritsis, Introduction to non-perturbative string theory, hep-th/9708130 [SPIRES].

[75] S. Giombi, A. Maloney and X. Yin, One-loop Partition Functions of 3D Gravity, JHEP 08 (2008) 007 [arXiv: 0804.1773] [SPIRES].

[76] J.R. David, M.R. Gaberdiel and R. Gopakumar, The Heat Kernel on AdS $S_{3}$ and its Applications, JHEP 04 (2010) 125 [arXiv:0911.5085] [SPIRES].

[77] R.B. Mann and S.N. Solodukhin, Universality of quantum entropy for extreme black holes, Nucl. Phys. B 523 (1998) 293 [hep-th/9709064] [SPIRES].

[78] J.A. Harvey and G.W. Moore, Fivebrane instantons and $R^{2}$ couplings in $N=4$ string theory, Phys. Rev. D 57 (1998) 2323 [hep-th/9610237] [SPIRES].

[79] D.V. Fursaev, Temperature and entropy of a quantum black hole and conformal anomaly, Phys. Rev. D 51 (1995) 5352 [hep-th/9412161] [SPIRES].

[80] R.B. Mann and S.N. Solodukhin, Conical geometry and quantum entropy of a charged Kerr black hole, Phys. Rev. D 54 (1996) 3932 [hep-th/9604118] [SPIRES]. 
[81] A.J.M. Medved, A comment on black hole entropy or why Nature abhors a logarithm, Class. Quant. Grav. 22 (2005) 133 [gr-qc/0406044] [SPIRES].

[82] D.N. Page, Hawking radiation and black hole thermodynamics, New J. Phys. 7 (2005) 203 [hep-th/0409024] [SPIRES].

[83] R. Banerjee and B.R. Majhi, Quantum Tunneling Beyond Semiclassical Approximation, JHEP 06 (2008) 095 [arXiv:0805.2220] [SPIRES].

[84] R. Banerjee and B.R. Majhi, Quantum Tunneling, Trace Anomaly and Effective Metric, Phys. Lett. B 674 (2009) 218 [arXiv:0808. 3688] [SPIRES].

[85] B.R. Majhi, Fermion Tunneling Beyond Semiclassical Approximation, Phys. Rev. D 79 (2009) 044005 [arXiv:0809.1508] [SPIRES].

[86] R.-G. Cai, L.-M. Cao and N. Ohta, Black Holes in Gravity with Conformal Anomaly and Logarithmic Term in Black Hole Entropy, JHEP 04 (2010) 082 [arXiv:0911.4379] [SPIRES].

[87] P.B. Gilkey, Invariance theory, the heat equation and the Atiyah-Singer index theorem, Publish or Perish Inc., U.S.A. (1984).

[88] D.V. Vassilevich, Heat kernel expansion: User's manual, Phys. Rept. 388 (2003) 279 [hep-th/0306138] [SPIRES].

[89] K. Kirsten and A.J. McKane, Functional determinants by contour integration methods, Annals Phys. 308 (2003) 502 [math-ph/0305010] [SPIRES].

[90] T. Hartman and L. Rastelli, Double-trace deformations, mixed boundary conditions and functional determinants in AdS/CFT, JHEP 01 (2008) 019 [hep-th/0602106] [SPIRES].

[91] F. Denef, S.A. Hartnoll and S. Sachdev, Black hole determinants and quasinormal modes, Class. Quant. Grav. 27 (2010) 125001 [arXiv:0908.2657] [SPIRES].

[92] R. Camporesi, Harmonic analysis and propagators on homogeneous spaces, Phys. Rept. 196 (1990) 1 [SPIRES].

[93] R. Camporesi and A. Higuchi, Spectral functions and zeta functions in hyperbolic spaces, J. Math. Phys. 35 (1994) 4217 [SPIRES].

[94] R. Camporesi, The Spinor heat kernel in maximally symmetric spaces, Commun. Math. Phys. 148 (1992) 283 [SPIRES].

[95] R. Camporesi and A. Higuchi, Arbitrary spin effective potentials in anti-de Sitter space-time, Phys. Rev. D 47 (1993) 3339 [SPIRES].

[96] S.N. Solodukhin, On 'Nongeometric' contribution to the entropy of black hole due to quantum corrections, Phys. Rev. D 51 (1995) 618 [hep-th/9408068] [SPIRES].

[97] S.N. Solodukhin, The Conical singularity and quantum corrections to entropy of black hole, Phys. Rev. D 51 (1995) 609 [hep-th/9407001] [SPIRES].

[98] B.S. DeWitt, Dynamical Theory of Groups and Fields, Gordon and Breach, New York U.S.A. (1965).

[99] H.P. McKean and I.M. Singer, Curvature and eigenvalues of the Laplacian, J. Diff. Geom. 1 (1967) 43 [SPIRES].

[100] S.M. Christensen and M.J. Duff, New Gravitational Index Theorems and Supertheorems, Nucl. Phys. B 154 (1979) 301 [SPIRES]. 
[101] S.M. Christensen and M.J. Duff, Quantizing Gravity with a Cosmological Constant, Nucl. Phys. B 170 (1980) 480 [SPIRES].

[102] A. Sen, Two Charge System Revisited: Small Black Holes or Horizonless Solutions?, JHEP 05 (2010) 097 [arXiv:0908.3402] [SPIRES].

[103] R. Camporesi and A. Higuchi, On the Eigen functions of the Dirac operator on spheres and real hyperbolic spaces, J. Geom. Phys. 20 (1996) 1 [gr-qc/9505009] [SPIRES].

[104] E. Witten, On S duality in Abelian gauge theory, Selecta Math. 1 (1995) 383 [hep-th/9505186] [SPIRES].

[105] M.J. Duff and P. van Nieuwenhuizen, Quantum Inequivalence of Different Field Representations, Phys. Lett. B 94 (1980) 179 [SPIRES].

[106] W. Siegel, Hidden Ghosts, Phys. Lett. B 93 (1980) 170 [SPIRES].

[107] J. Thierry-Mieg, Brs Structure of the Antisymmetric Tensor Gauge Theories, Nucl. Phys. B 335 (1990) 334 [SPIRES].

[108] E. Sezgin and P. van Nieuwenhuizen, Renormalizability Properties of Antisymmetric Tensor Fields Coupled to Gravity, Phys. Rev. D 22 (1980) 301 [SPIRES].

[109] I.L. Buchbinder, E.N. Kirillova and N.G. Pletnev, Quantum Equivalence of Massive Antisymmetric Tensor Field Models in Curved Space, Phys. Rev. D 78 (2008) 084024 [arXiv:0806.3505] [SPIRES].

[110] M.B. Green, J.H. Schwarz and E.Witten, Superstring Theory, Volume II: Loop Amplitudes, Anomalies And Phenomenology, Cambridge Monographs On Mathematical Physics, Cambridge University Press, Cambridge U.K. (1987).

[111] J. Maharana and J.H. Schwarz, Noncompact symmetries in string theory, Nucl. Phys. B 390 (1993) 3 [hep-th/9207016] [SPIRES].

[112] A. Sen, Strong - weak coupling duality in four-dimensional string theory, Int. J. Mod. Phys. A 9 (1994) 3707 [hep-th/9402002] [SPIRES].

[113] E. Witten, Analytic Continuation Of Chern-Simons Theory, arXiv:1001.2933 [SPIRES].

[114] S. Chaudhuri and D.A. Lowe, Type IIA heterotic duals with maximal supersymmetry, Nucl. Phys. B 459 (1996) 113 [hep-th/9508144] [SPIRES].

[115] P.S. Aspinwall, Some relationships between dualities in string theory, Nucl. Phys. Proc. Suppl. 46 (1996) 30 [hep-th/9508154] [SPIRES].

[116] S.M. Christensen, M.J. Duff, G.W. Gibbons and M. Roček, Vanishing One Loop $\beta$-function In Gauged N > 4 Supergravity, Phys. Rev. Lett. 45 (1980) 161 [SPIRES].

[117] J. Polchinski, Evaluation of the One Loop String Path Integral, Commun. Math. Phys. 104 (1986) 37 [SPIRES].

[118] O.A. Bedoya and N. Berkovits, GGI Lectures on the Pure Spinor Formalism of the Superstring, arXiv:0910.2254 [SPIRES].

[119] N. Berkovits, M. Bershadsky, T. Hauer, S. Zhukov and B. Zwiebach, Superstring theory on $A d S_{2} \times S^{2}$ as a coset supermanifold, Nucl. Phys. B 567 (2000) 61 [hep-th/9907200] [SPIRES].

[120] B. Chandrasekhar, Black Hole Partition Function using Hybrid Formalism of Superstrings, arXiv:0811.1758 [SPIRES] 